\title{
Fast Computational Aeroelastic Analysis of Helicopter Rotor Blades
}

\author{
Dominique Fleischmann*, Mudassir Lone ${ }^{\dagger}$ \\ Cranfield University, Cranfield, MK43 OAL, United Kingdom \\ Simone Weber $\$$ \\ Airbus Helicopters UK, Kidlington, OX5 1QZ, United Kingdom \\ Anuj Sharma II \\ BHR Group, The Fluid Engineering Centre, Cranfield, Bedfordshire MK43 OAJ, United Kingdom
}

The use of a new aeroelastic computer framework called Flexit is described and the framework is used to analyse the dynamic aeroelastic behaviour of a four-bladed helicopter main rotor. Flexit implements a loose coupling between unsteady vortex lattice method (UVLM) and numerical solution of the inhomogeneous Euler-Bernoulli partial differential equation (PDE). The framework is fast because most of the intensive computational functionality is performed on GPU using NVIDIA CUDA technology, and this makes it suitable for use in the early design stages. The UVLM algorithm uses a free wake model, and solution of the Euler-Bernoulli PDE is approximated using a finite difference algorithm that includes a term to take account of centrifugal forces. The results of simulations are compared with analysis performed with CFD and FSI tools.

\section{Introduction}

Today's push for rapid design development has highlighted the need for tools that are computationally cheap and technically versatile. These tools need to allow the broad exploration of design spaces and also provide insight into the physics governing the dynamics of the system being considered. Aeroelasticity is one such technical field where such a tool is needed. In this paper, the authors present a highly versatile tool for aeorelastic analysis called Flexit and demonstrate its capabilities for the time domain nonlinear aeroelastic simulation of helicopter rotor blades.

Aeroelastic analysis requires the application and integration of at least two engineering disciplines; aerodynamic loads prediction and structural analysis. Additional disciplines may include flight dynamics and control theory. For the aeroelastic investigation of fixed-wing aircraft, strip theory and doublet-lattice methods have traditionally been used for the prediction of aerodynamic loads, but there are various disadvantages to these approaches. For example, when the velocity field of the free wake directly affects the aeroelastic performance, and where empirical estimates of the velocity field are considered insufficient. For rotorcraft this is indeed the case; particularly so when the helicopter is hovering. One approach to predicting aerodynamic loads and the geometry and velocity profile of the free wake is the Vortex Lattice Method (VLM), and when geometric and kinematic non-linearities are to be taken into account, the Unsteady Vortex Lattice Method (UVLM). A good introduction to the UVLM can be found in reference [1] . A review of the application of UVLM in an aeroelastic setting and a discussion of the factors affecting the choice of analysis approach is presented in reference [2].

Analysis using even medium-fidelity calculations that take account of the wake has traditionally required significant resources and computing power and so in many settings it has only been performed during the latter stages of the design process. The need to rule out designs with unwanted aeroelastic properties at an early stage becomes more important when large dimensional parametric design spaces are being considered. It is therefore a benefit to have fast computational tools that can be used to analyse large numbers of preliminary designs quickly and discard any unsuitable designs before significant time and effort is wasted. The computational framework Flexit has been developed to address this need.

Flexit loosely couples UVLM computations for the prediction of unsteady aerodynamic loads and a finite difference approximation of the Euler-Bernoulli inhomogeneous PDE for the prediction of time-varying structural deformations.

\footnotetext{
* Research Fellow, Centre for Aeronautics, School of Aerospace, Transport \& Manufacturing, d.s.fleischmann@cranfield.ac.uk

${ }^{\dagger}$ Lecturer, Centre for Aeronautics, School of Aerospace, Transport \& Manufacturing, m.m.lone@cranfield.ac.uk

† Technology Integration Manager, Airbus Helicopters UK, simone .weber@airbus. com

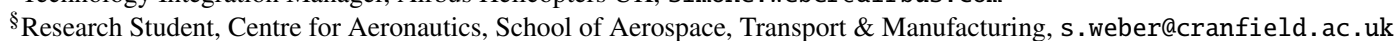

IIBHR Group, The Fluid Engineering Centre, Cranfield, Bedfordshire MK43 OAJ, United Kingdom ASharma@bhrgroup. com
} 
Very fast execution time is obtained on modest hardware by using NVIDIA CUDA technology to perform the computationally intensive work on the Graphical Processing Unit (GPU).

Throughout the conception, design and development of Flexit, we have adhered to a strict philosophy of avoiding the introduction of empirical corrections. The result is that, while certain physical phenomena such as viscous flow effects have been completely ignored, the use of Flexit as an objective comparison tool has not been compromised.

There are very many existing software packages, both commercial and open source, available that implement VLM and UVLM methods, and that can be used by aircraft designers. Some examples are XFoil (see reference [3]), OpenVOGEL (see reference [4]). In addition to fully-fledged mature software 'packages', there exist very many codes developed by industry and academia that are used for research and development. An example of such software is presented in reference [5] for the analysis of fish propulsion. This code has also been applied to the analysis of flapping wing bird flight in reference [6]. The number of packages available that integrate the (U)VLM method with a structural solver to allow aeroelastic analysis is somewhat less. To the knowledge of the authors, Flexit is the first integrated software package implementing UVLM with a structural solver that exploits the computational power of NVIDIA GPUs for computationally intensive calculations. Flexit has already been used with some success in the aeroelastic analysis of future concept designs of high aspect ratio passenger aircraft, and in this context, results have been qualitatively compared with the $\mathrm{CA}^{2} \mathrm{LM}$ framework (see reference [7]). Significant effort has already been spent in verifying grid convergence and stability of the numerical methods.

The aim of the work described in this paper is a) to demonstrate that the Flexit computational framework can be usefully applied to the aeroelastic analysis of helicopter main rotor designs, b) support the validation of the mathematical models implemented in the framework and c) predict the dynamic aeroelastic behaviour of the main rotor of the H135 helicopter design. Apart from the qualitative comparison with $\mathrm{CA}^{2} \mathrm{LM}$, most of the validation to date has been done by comparing results of computations with analytic results for very specific cases where analytic solutions exist, and the work here extends those highly simplified cases.

In this paper we begin with a brief outline of the theory implemented in the coupled aeroelastic tool Flexit. Next, we give an overview of the software, highlighting the implementation of the algorithms and we explain how we achieve a fast computational performance. After that we describe the simplifications and assumptions made in the Flexit analysis of the Airbus Helicopters H135 production helicopter rotor disk design. We follow this by presenting the results of the computations for the $\mathrm{H} 135$ under specific operating conditions. We continue with a comparison of the Flexit predictions with a variety of other prediction models. We discuss these comparisons, explain possible reasons for discrepancies and we conclude the paper by listing ideas for future work.

\section{Theory overview}

Flexit is a computer framework for performing time-domain unsteady aeroelastic analysis of aircraft during the early design stages. It implements an Unsteady Vortex Lattice Method (UVLM), a new method for numerical solution of the inhomogeneous Euler-Bernoulli partial differential equation (PDE) to predict the dynamic flapping deformation behaviour of the rotor blades under aerodynamic, structural deformation, centrifugal and inertial loads, and it implements a simple finite element analysis to predict the distribution of torsional twist along the span of each rotor blade.

For the computations described in this paper we only consider the aerodynamic behaviour and structural deformation of the main rotor blades in isolation.

The UVLM, Euler-Bernoulli and twist computations are performed alternately and repeatedly, moving forward in discrete time steps. The pressure distributions predicted by each UVLM calculation feeds into the external loading function used in the numerical solution of the Euler-Bernoulli equation and the aerodynamic pitching moment distribution feeds into the twist deformation distribution calculation. The resulting predictions of the instantaneous structural deformations are used to adjust the panel geometry for the next iteration of UVLM calculations. In this way, the structural deformation and aerodynamic load distributions are loosely coupled, and a discretely sampled prediction of the aerodynamic forces and dynamic deformation of the structure can be obtained.

The computational framework is implemented in $\mathrm{C}++$ and typically executes on a Windows desktop machine with CUDA-enabled hardware. The computation is fast because most of the intensive computations are performed on GPU using NVIDIA Compute Unified Device Architecture (CUDA) technology (see reference [8] for further information). 


\section{A. Applicability of the proposed analysis methods}

Before proceeding, it is important to note the domains of applicability of the UVLM and Euler-Bernoulli PDE and to check that application of the methods to the H135 helicopter are valid. The UVLM uses potential flow theory, and so assumes that fluid viscous forces are insignificant compared with intertia forces. This is typically the case for high Reynolds number attached flows. For a helicopter blade the Reynold's number is given by

$$
R e=\frac{\Omega R L}{v}
$$

where $\Omega$ is the angular velocity, $R$ is the radius, $L$ is the blade chord length, and $v$ is the kinematic viscosity. In the case of the H135 rotor disk, the lowest Reynold's number occurs at the location where the blade profile starts where the velocity is lowest. At this position on the blade we have a Reynolds number of approximately $1.2 \times 10^{6}$. This large value of $R e$ suggests that the flow over the blade aerofoil section is turbulent, and that viscous forces are small in comparison to inertia forces.

The UVLM also assumes incompressible flow, and so can only be applied to low speed flows significantly less than the speed of sound. The limiting case for this criteria is the speed of the flow at the rotor blade tip, where the flow speed is approximately $215 \mathrm{~m} / \mathrm{sec}$. Using a speed of sound at the operating altitude and temperature of $320 \mathrm{~m} / \mathrm{sec}$, we obtain a maximum hovering Mach number of approximately 0.68. Taking these Reynolds number and Mach number values, together with the assumption that the flow remains attached, we see that the helicopter falls within the range of applicability of the UVLM.

At this point, the reader should note that at high subsonic speeds it is necessary to include the Prandtl Glauert correction to take account of compressibility effects. Flexit does not include this correction and the effects of its exclusion are discussed in Section VII

The Euler-Bernoulli PDE assumes that the beam cross-sections do not deform out of plane. This restricts the approach to cases where the beam is long and slender, and where the magnitudes of transverse deformation are small compared with the beam length. For the case of the H135 rotor blade, it is certainly long and slender, and we know from observations of the rotor blades of the production helicopter during hovering flight that the maximum flapping deformations are of the order of $0.20 \mathrm{~m}$. Together, these observations lead us to conclude that the Euler-Bernoulli equation will give deformation predictions that are satisfactory at the early design stages.

\section{B. Unsteady vortex lattice method}

When applying the Flexit framework to the aeroelastic analyses of helicopters, the UVLM algorithm uses a free wake model to predict the downwash and the flow interaction between different lifting surfaces. This is particularly important when simulating hover flight because the trajectories of the blades pass very close to the wakes shed on previous rotations of the rotor disk. The implementation in Flexit loosely follows the Katz and Plotkin approach presented in reference [1]. The goal of VLMs is to determine the strength of vorticity $\Gamma$ on a set of $N$ finite vortex line segments distributed in a topologically rectangular grid of panels over a representation of the lifting surfaces such that the flow velocity field satisfies Laplace's equation and such that certain boundary conditions are met. In physical terms these boundary conditions are that the flow velocity is always tangential to all the lifting surfaces everywhere, and that the magnitude of the perturbation velocity tends to zero as the distance from the lifting surfaces tends to infinity. On each panel in the grid, these boundary conditions can be stated formally as:

$$
(\nabla \phi-\vec{w}(x, y, z, t)) \cdot \vec{n}(x, y, z, t)=0, \text { for }(x, y, z) \in \partial D
$$

and

$$
\nabla \phi \rightarrow 0, \text { as } x^{2}+y^{2}+z^{2} \rightarrow \infty
$$

where $\nabla$ is the Laplacian operator, and where $\nabla \phi=(\nabla \phi)(x, y, z, t)$ is the time-varying perturbation velocity vector field induced by the vortex segments on the surfaces (of a priori unknown strength), and $\vec{w}(x, y, z, t)$ is the known free-stream velocity, and where $\vec{n}(x, y, z, t)$ is the time-varying local unit normal vector to the lifting surfaces, and where $\partial D$ is the set of points representing the lifting surface. In the context of this aeroelastic analysis, $\vec{w}(x, y, z, t)$ includes the velocities due to vortices shed into the wake of the lifting surfaces as the surfaces move though the fluid, any time-varying morphing velocities of the surface, the rigid body motion of the vehicle, the deflection of any control surfaces, and any 
a priori known externally applied gust velocity field. Finally, we note that $\vec{n}(x, y, z, t)$ takes into account rigid body motion, any control surface deflections and local angular displacements due to structural deformations.

The sets of equations (2) and (3) (one for each panel in the grid) are manipulated to form a system of simultaneous equations in the unknown strengths of each of the vortex line segments in the grid representing the lifting surfaces. The strengths of the vortex line segments $\Gamma$, known as the "vorticities", are scaling factors of vector velocity field functions due to each of the vortices. In traditional UVLM implementations the Biot-Savart formulation is used for the vector velocity field functions. The Flexit framework has an option to implement either the Biot-Savart formulation, or a Rankine vortex velocity field function. The Rankine formulation introduces more computational complexity but has the advantage of avoiding unbounded velocity magnitudes that are predicted by the Biot-Savart formulation as one approaches close to the vortex line segment. We comment further on this in section VIII

\section{Euler-Bernoulli equation finite difference method}

The dynamic behaviour of each main rotor blade of span $L$ is modelled using the inhomogeneous Euler-Bernoulli partial differential equation with damping and centrifugal force terms, and is given in reference [9] as

$$
\begin{aligned}
\frac{\partial^{2}}{\partial t^{2}} w(x, t)= & \frac{q(x, t)}{\mu(x)} \\
& -\frac{1}{\mu(x)} \frac{\partial^{2}}{\partial x^{2}}\left(E(x) I(x) \frac{\partial^{2}}{\partial x^{2}} w(x, t)\right) \\
& +\frac{1}{\mu(x)} \frac{\partial}{\partial x}\left(T(x) \frac{\partial}{\partial x} w(x, t)\right) \\
& -\beta \frac{\partial}{\partial t} w(x, t),
\end{aligned}
$$

where $w: \mathbb{R}^{+} \times[0, L] \rightarrow \mathbb{R}$ is the apriori unknown transverse (flapping) deformation function of time and distance along the beam, $q: \mathbb{R}^{+} \times[0, L] \rightarrow \mathbb{R}$ is the known function describing the external load (in this case the aerodynamic lift distribution along the span), $\mu:[0, L] \rightarrow \mathbb{R}$ is the distribution of the mass per unit length along the beam, $E:[0, L] \rightarrow \mathbb{R}$ is the distribution of material stiffness (Young's modulus) along the length of the beam, $I:[0, L] \rightarrow \mathbb{R}$ is the distribution of second moment of area of the beam about the beam flapping axis and $\beta \in \mathbb{R}^{+}$is a deformation velocity-dependent damping coefficient. The exact relationship between the velocity dependent damping coefficient $\beta$ used in this work and the Rayleigh damping used in other structural simulation work is a topic that will require further investigation. We include this as an item of future work in section VIII] The function $T:[0, L] \rightarrow \mathbb{R}$ accounts for the effect of centrifugal force and is given by:

$$
T(x)=\int_{x}^{L} \mu(u) \Omega^{2}(R+u) d u,
$$

where, as before, $L$ is the radius of the tip of the blade beam, and $R$ is the radius of the root of the blade beam, $\Omega$ is the angular velocity of the rotor blade and $u$ is a dummy variable of integration. Note that for the helicopter main rotor configuration considered here, the part of the blade that produces aerodynamic forces extends from the blade tip to a point some distance outboard of the rotor hub, while the beam being modelled extends from the tip all the way to the hub.

Flexit framework uses a novel finite difference algorithm to approximate the solution of the Euler-Bernoulli PDE at discretely sampled time steps and span-wise positions along the length of each of the four main rotor blades. The finite 
difference expression based on equation (4) used to predict an approximate value of $w\left(x_{i}, t_{n+1}\right)$ is given by

$$
\begin{aligned}
w\left(x_{i}, t_{n+1}\right) & \approx \frac{1}{1+\frac{\beta \Delta t}{2}}\left[\frac{(\Delta t)^{2}}{\mu\left(x_{i}\right)} q\left(x_{i}, t_{n}\right)\right. \\
& -\frac{(\Delta t)^{2}}{\mu\left(x_{i}\right)} S\left(x_{i}, t_{n}\right) \\
& +(\Delta t)^{2} D\left(x_{i}, t_{n}\right) \\
& +\left(\frac{\beta \Delta t}{2}-1\right) w\left(x_{i}, t_{n-1}\right) \\
& \left.+2 w\left(x_{i}, t_{n}\right)\right]
\end{aligned}
$$

where

$$
\begin{aligned}
S\left(x_{i}, t_{n}\right) & \approx \frac{1}{(\Delta x)^{4}}\left\{-\frac{1}{12} F\left(x_{i-1}\right) w\left(x_{i-4}, t_{n}\right)+\frac{1}{3} F\left(x_{i-1}\right) w\left(x_{i-3}, t_{n}\right)+\left[\frac{1}{2} F\left(x_{i-1}\right)+\frac{1}{6} F\left(x_{i}\right)\right] w\left(x_{i-2}, t_{n}\right)\right. \\
& +\left[-\frac{5}{3} F\left(x_{i-1}\right)-\frac{8}{3} F\left(x_{i}\right)\right] w\left(x_{i-1}, t_{n}\right)+\left[\frac{11}{12} F\left(x_{i-1}\right)+5 F\left(x_{i}\right)+\frac{11}{12} F\left(x_{i+1}\right)\right] w\left(x_{i}, t_{n}\right) \\
& +\left[-\frac{8}{3} F\left(x_{i}\right)-\frac{5}{3} F\left(x_{i+1}\right)\right] w\left(x_{i+1}, t_{n}\right)+\left[\frac{1}{6} F\left(x_{i}\right)+\frac{1}{2} F\left(x_{i+1}\right)\right] w\left(x_{i+2}, t_{n}\right) \\
& \left.+\frac{1}{3} F\left(x_{i+1}\right) w\left(x_{i+3}, t_{n}\right)-\frac{1}{12} F\left(x_{i+1}\right) w\left(x_{i+4}, t_{n}\right)\right\}
\end{aligned}
$$

and

$$
F\left(x_{i}\right)=E\left(x_{i}\right) I\left(x_{i}\right)
$$

and

$$
\begin{aligned}
& D\left(x_{i}, t_{n}\right)= \\
& \frac{\Omega^{2}}{\mu\left(x_{i}\right)} R\left(\frac{-\frac{1}{12} w\left(x_{i-2}, t_{n}\right)+\frac{4}{3} w\left(x_{i-1}, t_{n}\right)-\frac{5}{2} w\left(x_{i}, t_{n}\right)+\frac{4}{3} w\left(x_{i+1}, t_{n}\right)-\frac{1}{12} w\left(x_{i+2}, t_{n}\right)}{(\Delta x)^{2}}\right) I_{1}\left(x_{i}, t_{n}\right) \\
& +\frac{\Omega^{2}}{\mu\left(x_{i}\right)} R\left(\frac{w\left(x_{i+1}, t_{n}\right)-w\left(x_{i-1}, t_{n}\right)}{2 \Delta x}\right)\left(\frac{I_{1}\left(x_{i+1}, t_{n}\right)-I_{1}\left(x_{i-1}, t_{n}\right)}{2 \Delta x}\right) \\
& +\frac{\Omega^{2}}{\mu\left(x_{i}\right)}\left(\frac{-\frac{1}{12} w\left(x_{i-2}, t_{n}\right)+\frac{4}{3} w\left(x_{i-1}, t_{n}\right)-\frac{5}{2} w\left(x_{i}, t_{n}\right)+\frac{4}{3} w\left(x_{i+1}, t_{n}\right)-\frac{1}{12} w\left(x_{i+2}, t_{n}\right)}{(\Delta x)^{2}}\right) I_{2}\left(x_{i}, t_{n}\right) \\
& +\frac{\Omega^{2}}{\mu\left(x_{i}\right)}\left(\frac{w\left(x_{i+1}, t_{n}\right)-w\left(x_{i-1}, t_{n}\right)}{2 \Delta x}\right)\left(\frac{I_{2}\left(x_{i+1}, t_{n}\right)-I_{2}\left(x_{i-1}, t_{n}\right)}{2 \Delta x}\right)+O\left((\Delta x)^{2}\right),
\end{aligned}
$$

where

$$
I_{1}\left(x_{i}, t_{n}\right)=\int_{x_{i}}^{L} \mu(u) d u
$$

and

$$
I_{2}\left(x_{i}, t_{n}\right)=\int_{x_{i}}^{L} u \mu(u) d u .
$$

The approximate symbol " $\approx$ " in equations (6) and (7) is used because the terms of order $\Delta x$ and $\Delta t$ have been ignored.

Equation (7) is used to predict the value of $w$ at each span-wise position $x_{i}$ at the next time level $t_{n+1}$ based on predictions of $w$ at previous time steps and at adjacent span-wise positions. The finite difference stencil is illustrated in figure 1 . 


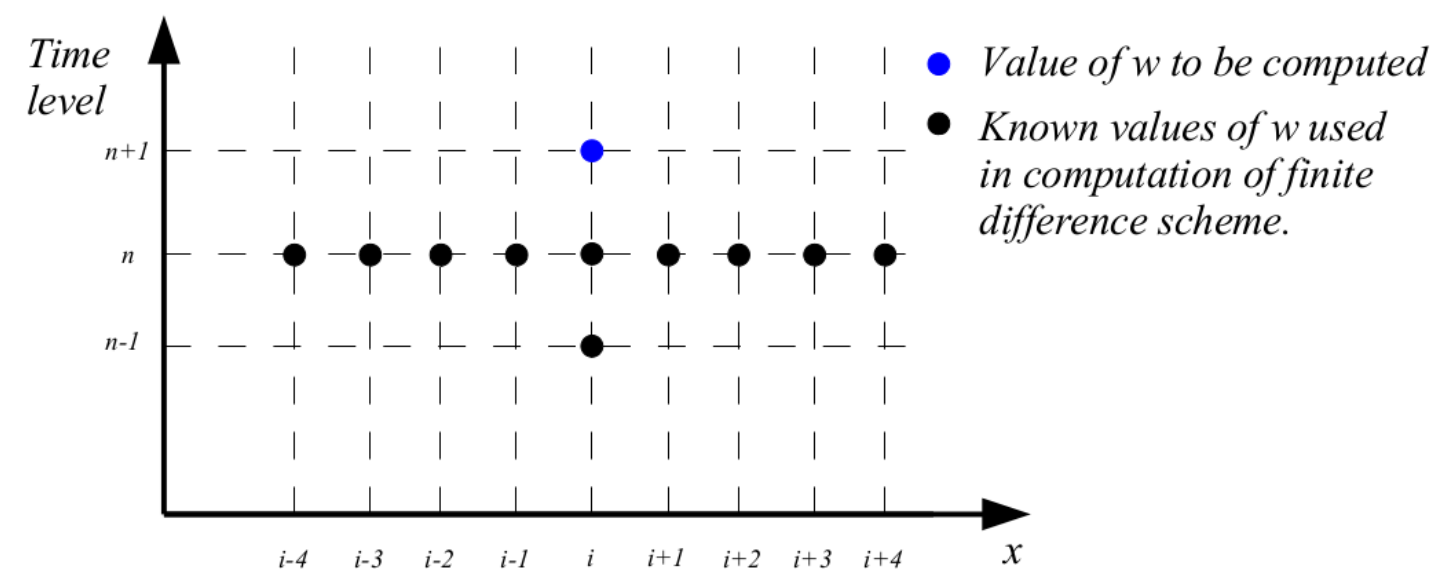

Fig. 1 Stencil for the finite difference scheme.

\section{Torsion finite difference scheme}

We model the dynamic torsional deformation of each rotor blade beam with a finite sequence of small beam elements placed end-to-end with the properties of each element approximating the average properties of the real beam at that location.

We consider a total of $N$ beam elements indexed by the subscript $i$ and corresponding to the $N$ nodes considered in the Euler-Bernoulli analysis described above. The externally applied torques at the left and right end of the element at time $t_{n}$ of the simulation for each $x_{i} \in\{1 \ldots N\}$ are denoted by $T^{L}\left(x_{i}, t_{n}\right)$ and $T^{R}\left(x_{i}, t_{n}\right)$ respectively. The arrangement of the beam elements is illustrated in figure 2 .

\section{$\begin{array}{lll}\text { Element } i-1 & \text { Element } i & \text { Element } i+1\end{array}$}
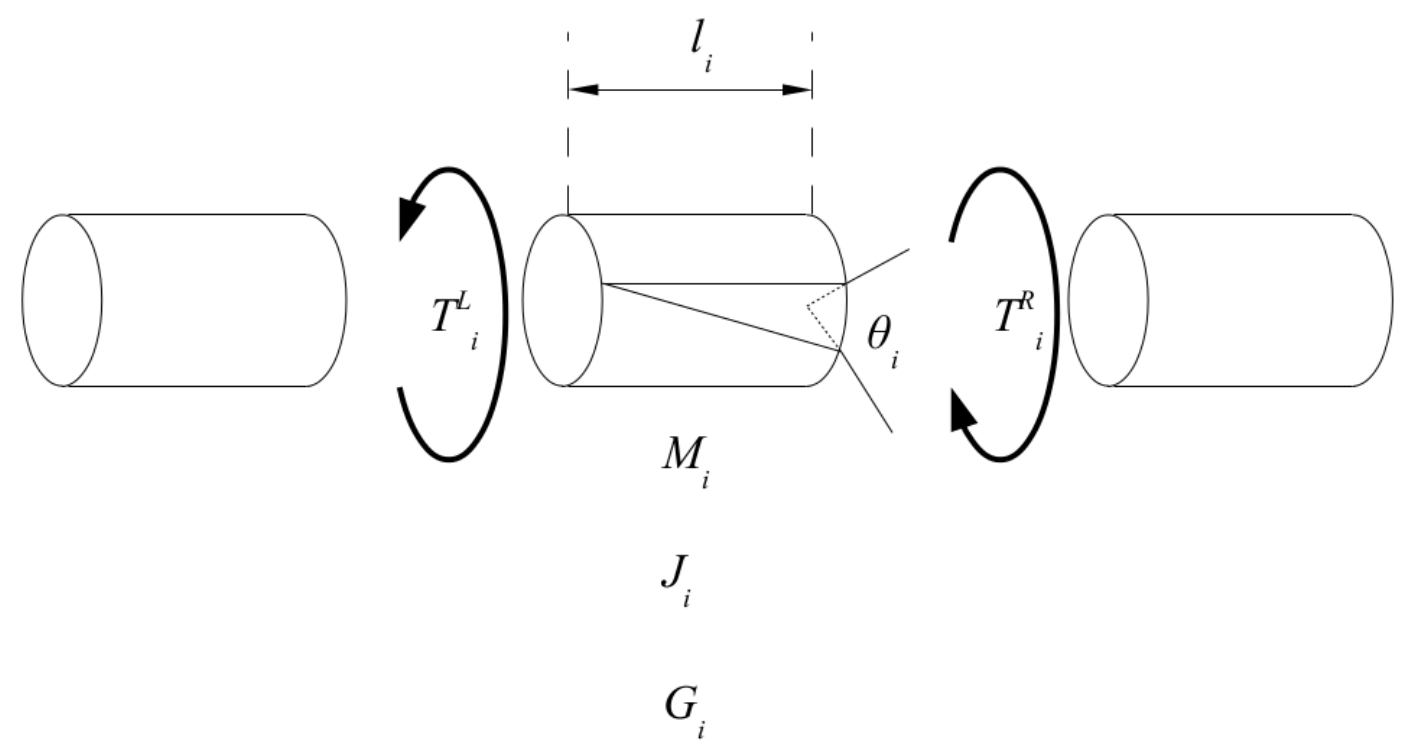

Fig. 2 Torsion beam elements.

At each Euler-Bernoulli beam node and at each time level, we express the torque $T^{\text {internal }}\left(x_{i}, t_{n}\right)$ due to the torsional twist strain angle $\theta\left(x_{i}, t_{n}\right)$ along the element between $x_{i}$ and $x_{i+1}$ as

$$
T^{\text {internal }}\left(x_{i}, t_{n}\right)=\frac{\theta\left(x_{i}, t_{n}\right) J_{i} G_{i}}{l_{i}},
$$


where $J_{i}$ is the polar second moment of area of the beam cross-section, where $G_{i}$ is the shear stress, and where $l_{i}$ is the length of the $i^{t h}$ finite element. The net torque applied to each element is then given by

$$
T^{n e t}\left(x_{i}, t_{n}\right)=\left(T^{L}\left(x_{i}, t_{n}\right)-T^{R}\left(x_{i}, t_{n}\right)\right)-T^{\text {internal }}\left(x_{i}, t_{n}\right) .
$$

The angular acceleration of each element at time $t_{n}$ is then

$$
\frac{\delta \omega_{i}}{\delta t}=\frac{T^{n e t}\left(x_{i}, t_{n}\right)}{I_{i}}
$$

where $I_{i}$ is the moment of inertia of the $i^{\text {th }}$ beam element in the torque rotation axis.

To determine the total angle of rotation of the beam element at time $t+\delta t$, we use numerical integration in two stages; first to find the angular velocity, and then to find the rotation angle. An Euler integration scheme is adopted for this work, so that the angular velocity of each element is given by

$$
\omega\left(x_{i}, t_{n+1}\right)=\omega\left(x_{i}, t_{n}\right)+\left.\Delta t \frac{\delta \omega_{i}}{\delta t}\right|_{t=t_{n}},
$$

and the angular strain rotation angle of each element is given by

$$
\theta\left(x_{i}, t_{n+1}\right)=\theta\left(x_{i}, t_{n}\right)+K_{T} \Delta t \omega\left(x_{i}, t_{n+1}\right),
$$

where $K_{T} \in \mathbb{R}$ is an angular velocity damping coefficient that generally satisfies $0<K_{T}<1$.

\section{Flexit software implementation and performance}

The Flexit computational framework comprises a total of seven executables written from scratch in $\mathrm{C}++$ that run under Windows 7. Six of these programs are used to perform pre- and post-processing and the seventh, itself called Flexit, actually does the aero-elastic computations. The Flexit program can be run interactively with a graphical user interface or as a batch program from the command line. Figure 3 presents a screenshot of the Flexit program graphical user interface.

An analysis work-flow consists of the user running each program in a prescribed sequence, being led through the process of defining the lifting surface geometry, describing the properties and connectivity of the Euler-Bernoulli beams, describing the kinematic motion of the lifting surfaces, actually doing the aeroelastic analysis, and finally postprocessing the results. Data output from the first program forms the input to the next, and so on. The Javascript Simple Object Notation (JSON) file format is used throughout. As well as the JSON files, functionality is also implemented to output data from the Flexit $\mathrm{C}++$ program as Matlab ${ }^{\circledR}$ script files with data arrays and script commands automatically included. Many of the figures in this paper have been prepared using this functionality.

The Flexit program performs the computationally intensive processing of the aeroelastic analysis. Most of the processing is concerned with doing the UVLM computations, and the most intensive part of this is concerned with computing the flow induced by the panels of known vorticity that have been shed into the wake. Fortunately the theory is amenable to parallel computation, and we have exploited this property to implement the algorithms in parallel on the GPU using the NVIDIA CUDA technology. In this way many thousands of computations are arranged to execute in parallel so that the overall elapsed time can be reduced by several orders of magnitude over a conventional CPU implementation. Each analysis case presented in this paper involves computation over 160 UVLM time steps with 640 bound panels and over 5000 free wake panels. On a DELL Precision M6800 mobile workstation with a single NVIDIA Quadro K5100M GPU with relatively modest performance each run took approximately 90 seconds of wall-clock time to complete.

Figure 4 presents a graph from a typical simulation performed during the preparation of this paper on the DELL Precision M6800 mobile workstation with the single NVIDIA Quadro K5100M GPU. It shows the wall-clock time for each computational frame, and the accumulated wall-clock time as the simulation progresses. The sudden increase in frame execution time at frame 155 may be related to GPU bandwidth, and is being investigated using code profiling techniques. Please see section VIII for further discussion of this topic. Figure 5 presents a corresponding area chart indicating the wall-clock time for each of the major computational tasks within a frame. From this figure it is clear that 


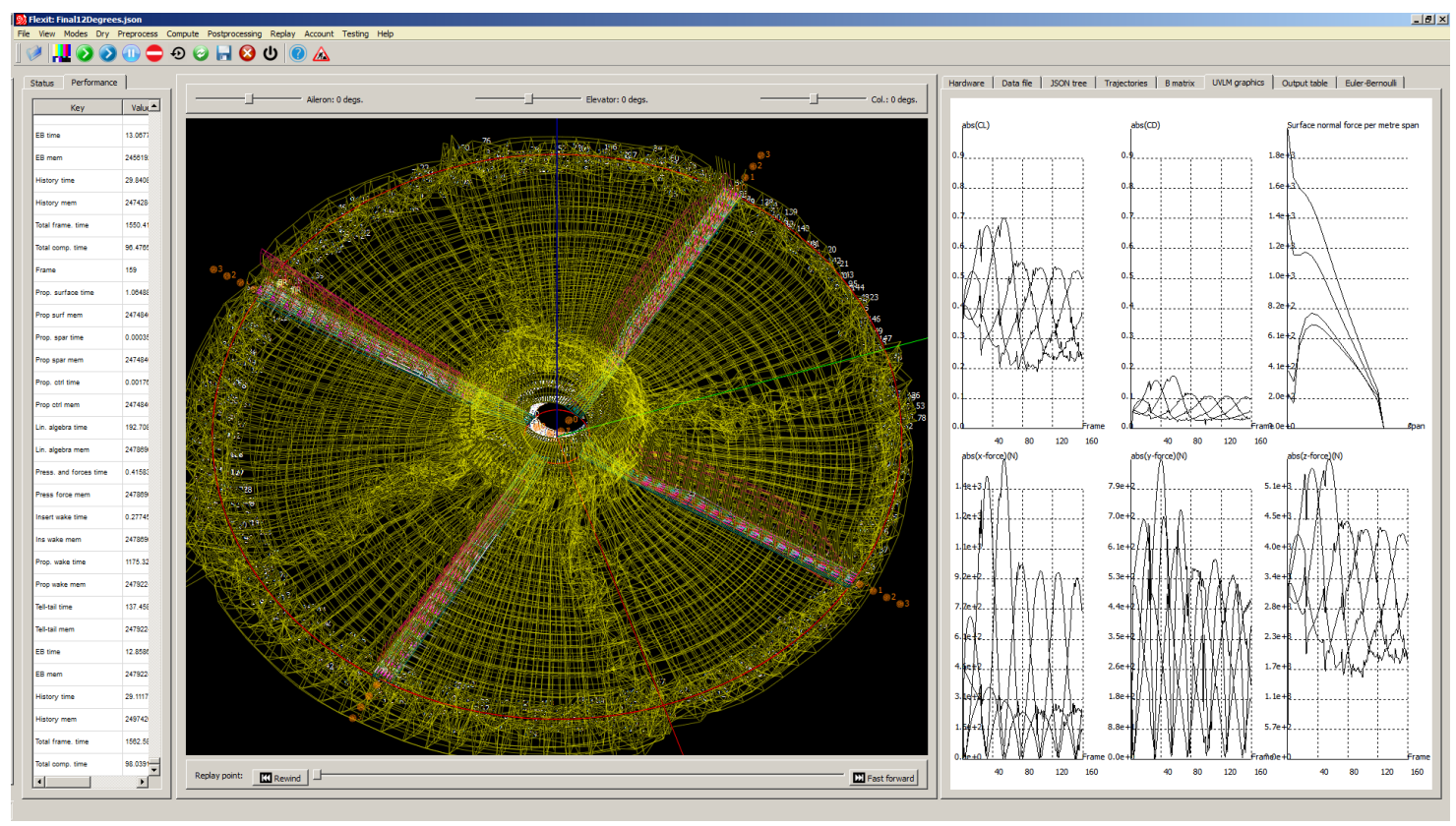

Fig. 3 Flexit screenshot

all computational tasks experience a small increase in computation time at frame 155, but it is the free wake propagation computation that is largely responsible for this increase in overall execution time.

The authors believe there still remain many ways to significantly improve computational performance by parallelizing other parts of the algorithm, and we intend to expend effort on this in the future.

\section{General Model Parameters}

One of the aims of the work presented in the paper is to predict the dynamic aeroelastic behaviour of the main rotor of the existing H135 helicopter, which is part of the Airbus Helicopters family. Figure 6/(reproduced from reference [10]) presents a general arrangement and principle particulars of the helicopter being studied.

The main rotor configuration is a bearingless main rotor (BMR) which is characterised by the lack of mechanical flapping, lead-lag and torsional hinges. The rotor blades consist of the Flexbeam, pitch control cuff, blade aerofoil section and an elastomeric lead-lag damper. The Flexbeam is designed such that the three required hinges are incorporated within the structure. A more detailed description of this rotor blade design can be found in reference [11] and reference [12] .

A certain amount of preparatory work is required to derive from the actual production helicopter design a set of numerical parameters suitable for input to the computation algorithm. In the next section we describe the factors taken into account in pre-processing the production design information to provide the input data for Flexit.

\section{Preparation of Flexit simulation input data}

We have used the manufactured aerofoil section, blade twist, material properties, flapping stiffness, blade mass and torsional stiffness as a function of blade span position provided by Airbus Helicopters Deutschland (AHD). The aeroelastic analysis was performed for hovering flight at an altitude of 1000 feet out of ground effect. Using the ideal gas equation, this altitude gives an air density of $1.18965 \mathrm{~kg} / \mathrm{m}^{3}$ and this value was used in all simulation runs for the computation of pressures and forces. Since the UVLM method does not implement sources or sinks, it is not possible to model bodies with internal volumes. Accordingly, we ignore the fuselage and tail boom and we ignore the thickness of the rotor blade sections. The production aircraft blade section camber has been derived from the aerofoil section and we place the UVLM panels along this slightly curved camber line. We use seven panels in the chord-wise direction (and 20 panels in the span-wise direction). This discretization scheme gives a compromise between modelling fidelity and computation time, while ensuring a roughly square panel geometry for the panels shed into the free wake. The actual 
Flexit overall computational performance H135 Cambered blades, 8 degree blade root pitch angle

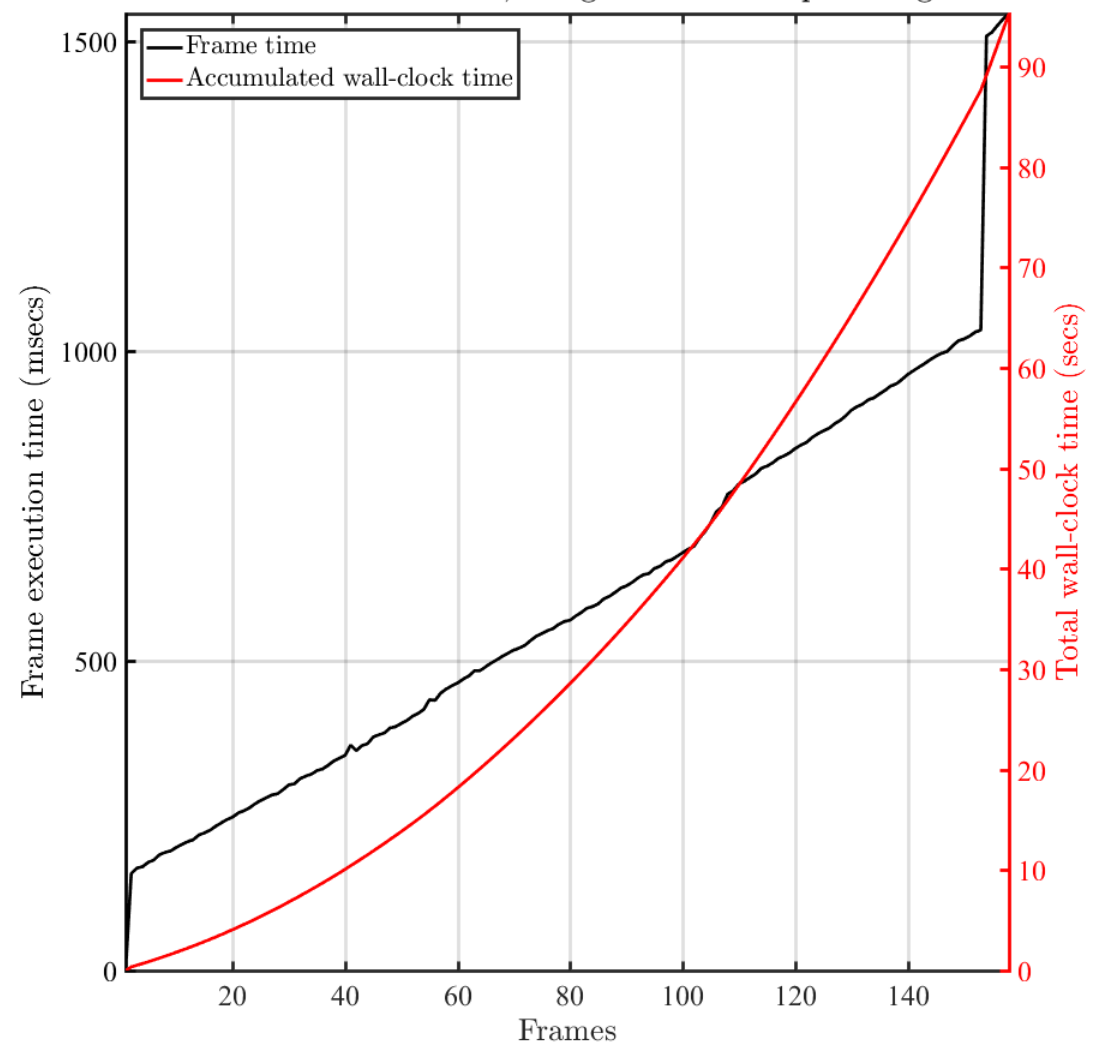

Fig. 4 Overall computational performance

production rotor blade features a curved leading edge planform shape near the tip. In order to simplify the analysis, the blade geometry used in the Flexit simulations assumes that the full chord profile extends right out to the tip as illustrated in figure 7. Note that figure 7 also indicates the cartesian coordinate system used in the Flexit analysis.

Blade mass distribution and flapping stiffness data has been linearly interpolated to generate the values of these variables at the 20 beam node positions. Note that we have been careful to arrange the beam node positions so that each node directly corresponds to exactly one chord-wise strip of UVLM panels. This makes it very simple to distribute UVLM panel forces on to the beam nodes. In general, the Flexit framework does not require this close correspondence.

The panel geometry of each blade in the UVLM analysis was generated from a Bezier surface patch, with six rows of control points along the span and five columns of control points along the chord. Due to the smoothing effect of Bezier curves and surfaces, placing the control points coincident with the production design twist distribution results in an erroneous panel twist distribution. To overcome this error, the production design blade pitch was approximated using manual curve fitting techniques. The inboard end of the H135 blade structural design (where there is no aerofoil section) is arranged so that flapping deformation and torsional deformation is accommodated by strain deflection in the blade beam. We have modelled the whole length of the blade from the rotor hub (at a radius of $0.47 \mathrm{~m}$ ) to the aerodynamic tip (at radius $5.2 \mathrm{~m}$ ) with beam elements that are free to deform in flapping and in torsion. Only the outboard section of the beam that corresponds to the aerodynamic blade section is subjected to aerodynamic loading from the UVLM computations.

The presence of the fuselage and tail boom have been ignored in the Flexit simulations. This has been done because the UVLM cannot model the presence of solid volumes. Since the fuselage has been ignored, the circumferential orientation of the four rotor blades can be arbitrarily assigned. Despite this, much of the simulation output material presented here identifies the blade numbers for convenience. The working helicopter tail rotor has been ignored in the Flexit simulations. This is simply to reduce computational complexity. 


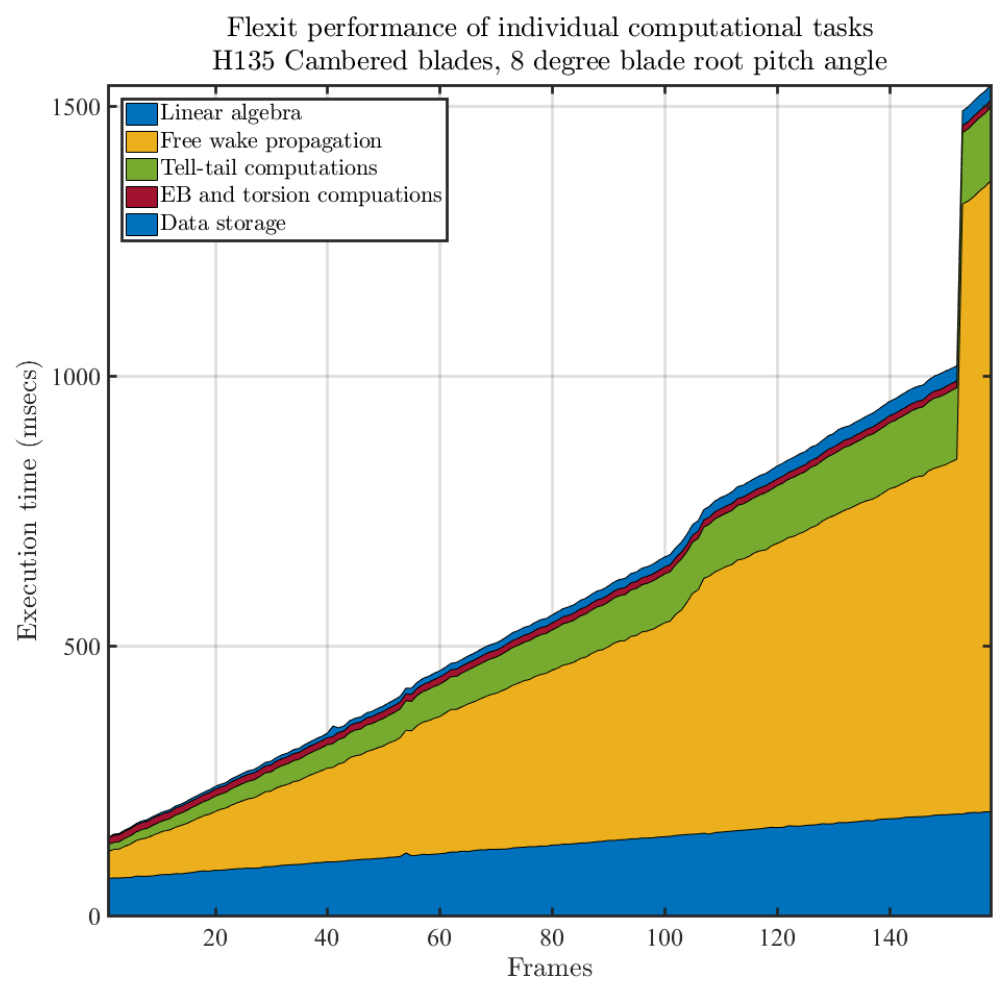

Fig. 5 Performance of individual computational tasks

In the hover, all forces and moments on the real helicopter are in equilibrium. Asymmetric fuselage drag from the main rotor downwash and a small vertical component of tail rotor forces cause small pitching and rolling moments that must be counteracted by equal and opposite forces and moments from the main rotor. This is achieved by tilting the main rotor slightly out of the horizontal. This small tilt angle has been ignored in the Flexit simulations and the main rotor tip path plane (before blade deformations are added) has been set up to be exactly horizontal in the earth frame.

Figure 8 indicates the arrangement of structural nodes and aerodynamic panels on rotor blades used in the Flexit simulations.

The elastic axis in the simulations has been set up at an arbitrary position of $45 \%$ of the chord length behind the leading edge. The exact location of the aero-centre is dependent on many factors including the inflow velocity profile, but in general it will be around 25\% of the chord length behind the leading edge. This difference in longitudinal position results in a small pitch-up moment arm. The result is that, in general, the blade will twist during flight so the outboard end of the blade will experience a larger angle of attack than the manufacturing jig twist value.

The Euler-Bernoulli finite difference scheme and the torsional deformation finite element scheme include velocitydependent damping terms. The bending and twist deformation dynamics are sensitive to the choice of the damping coefficients. For the Flexit analysis presented in this paper we have used a value of 20 for the Euler-Bernoulli damping coefficient $\beta$ (see the last term on the right-hand side of equation (4) and a value of 0.03 for the torsion deformation damping coefficient $K_{T}$.

\section{Results}

The Flexit program is configured so that all structural node and all UVLM panel state parameters can be recorded at each simulation time step, and output to files at the end of the simulation. This allows the user to review a great variety of output data. We present below a small sample of the results available from the software. We refer to the value of the angle of attack of the undeformed blade at the inboard end of the aerodynamic profile as the blade root pitch angle. Three design cases were analysed for the three blade root pitch angles of 8,10 and 12 degrees, and these three cases correspond to three collective control settings. 


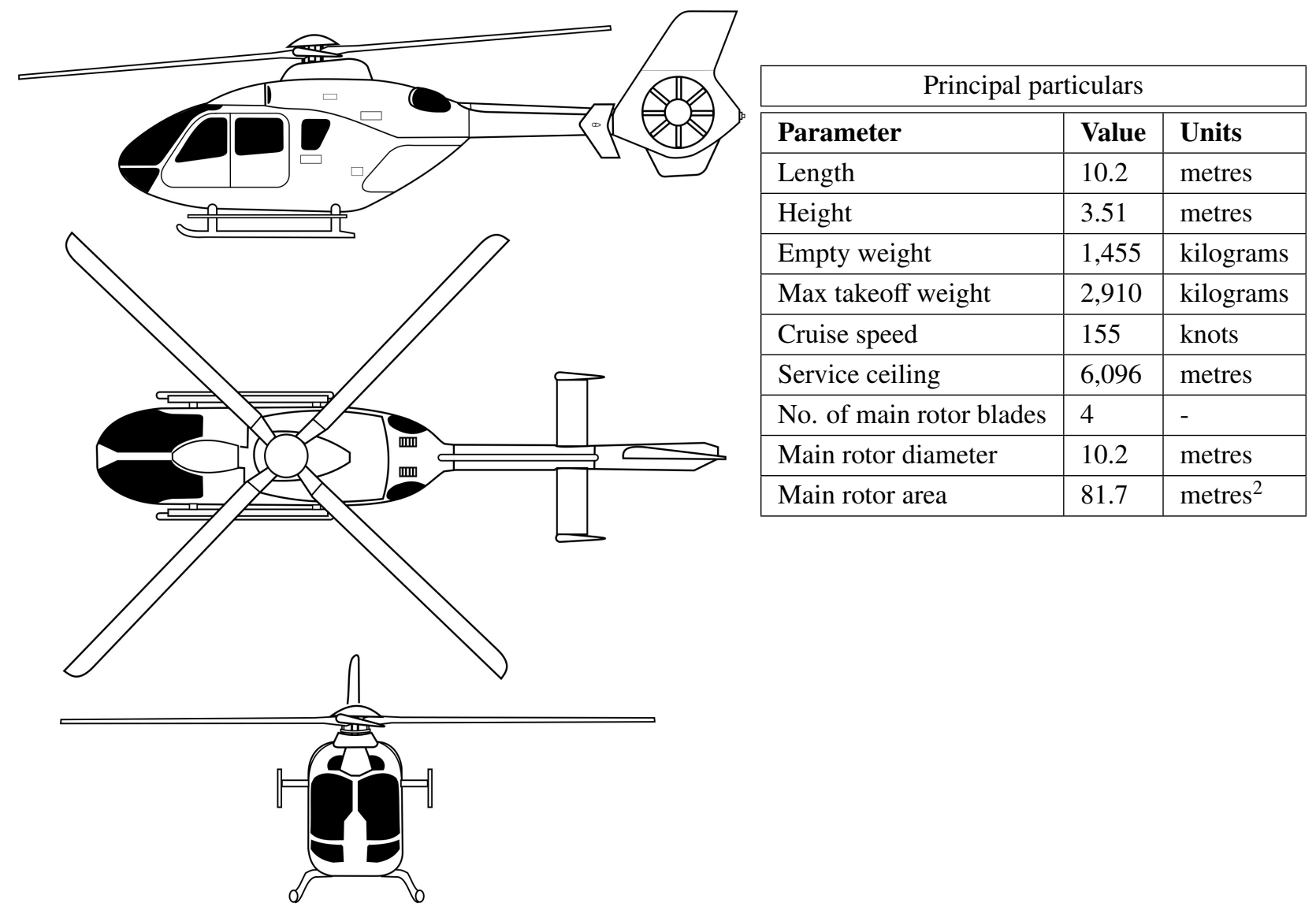

Fig. 6 H135 Helicopter general arrangement 


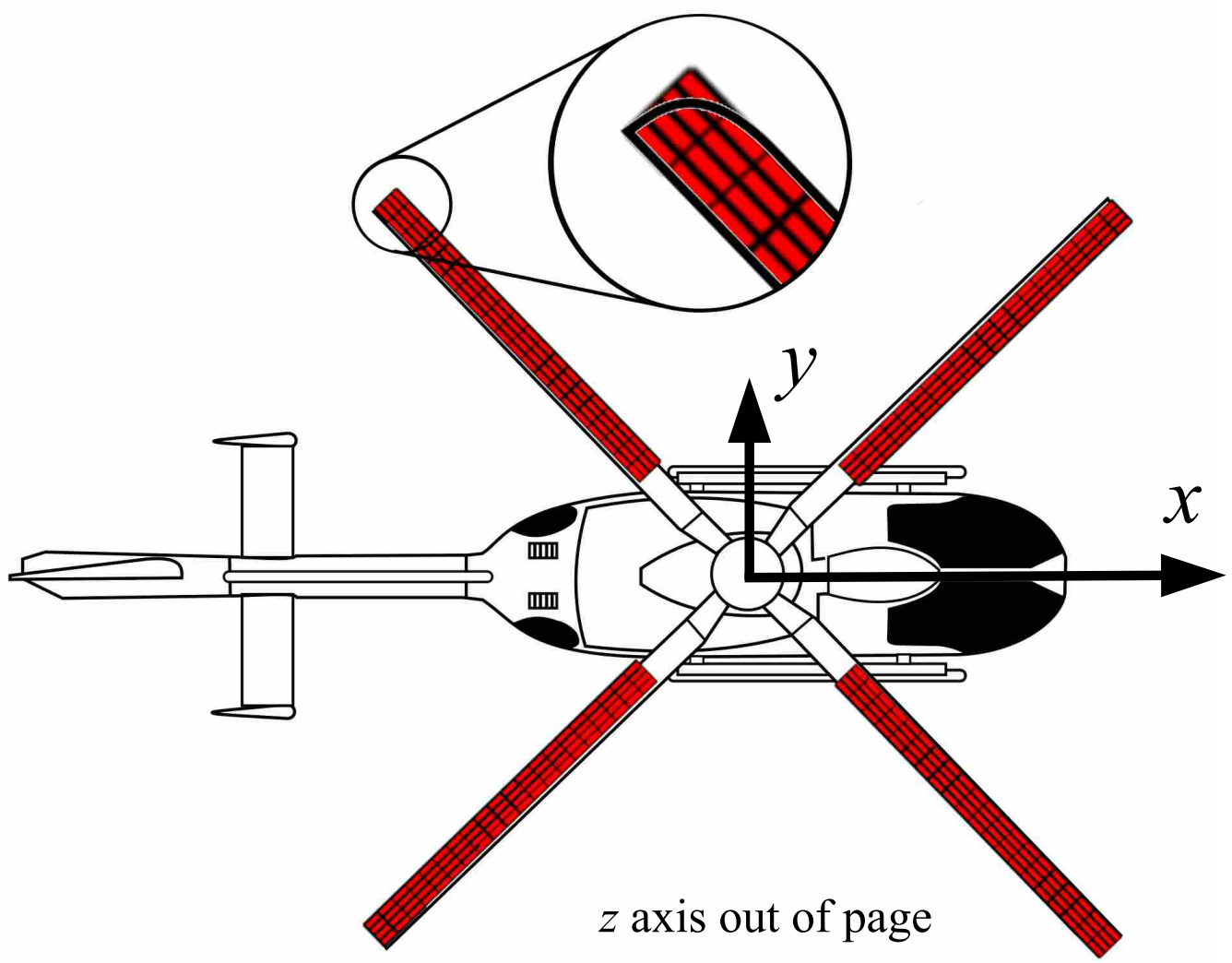

Fig. 7 Main rotor blade tip planform

A. Thrust coefficients

We define the thrust coefficient

$$
C_{T}=\frac{T}{\rho(\Omega R)^{2} \pi R^{2}},
$$

as given in reference [13], where $T$ is the total vertical aerodynamic force due to all four blades acting at the centre of the hub, $\rho$ is the air density, $\Omega$ is the angular velocity of the blades and $R$ is the radius of the blade tips. Figure 9 presents the predicted thrust coefficient $C_{T}$ and the blade torsional twist deformations as a function of time (computational frame number) for a range of blade root twist angles.

The lift produced by the blades rises rapidly at the start of the simulation as the starting vortex is shed from the trailing edge of each blade and the vorticity builds up. Then, at around frame 25 when the blades have rotated a quarter of a revolution, the blades start to move past the wake left behind by the blade in front. Encountering this downwash causes a sudden reduction in angle of attack. At the same time, the torsional deformation rises, causing an increase in the angle of attack. To a certain extent these two effects offset each other. At approximately frame 100 when the blades have performed just over one revolution and have passed through several wakes of blades ahead, the initial transient large torsional deformation has begun to die down, and the effective angle of attack drops, causing the thrust coefficient settle at a steady reduced value.

\section{B. Pressure distributions}

When the simulation starts there is no down-wash so the values of the lift forces are large. As the simulation progresses, the down-wash experienced by the blades increases and as a result the angle of attack decreases. The angle of attack at the outboard end of the blade at the end of the simulation (when the down-wash is fully developed) is about the same magnitude as the blade twist angle so the net lift at the tip gets very small. The result is that Flexit predicts a negative pressure at the tip on the leading edge. This can be seen in figure 10 where the leading edge pressure difference at the tip is negative. Note that the red mesh in figure 10 indicates the surface of the blade modelled in Flexit. 


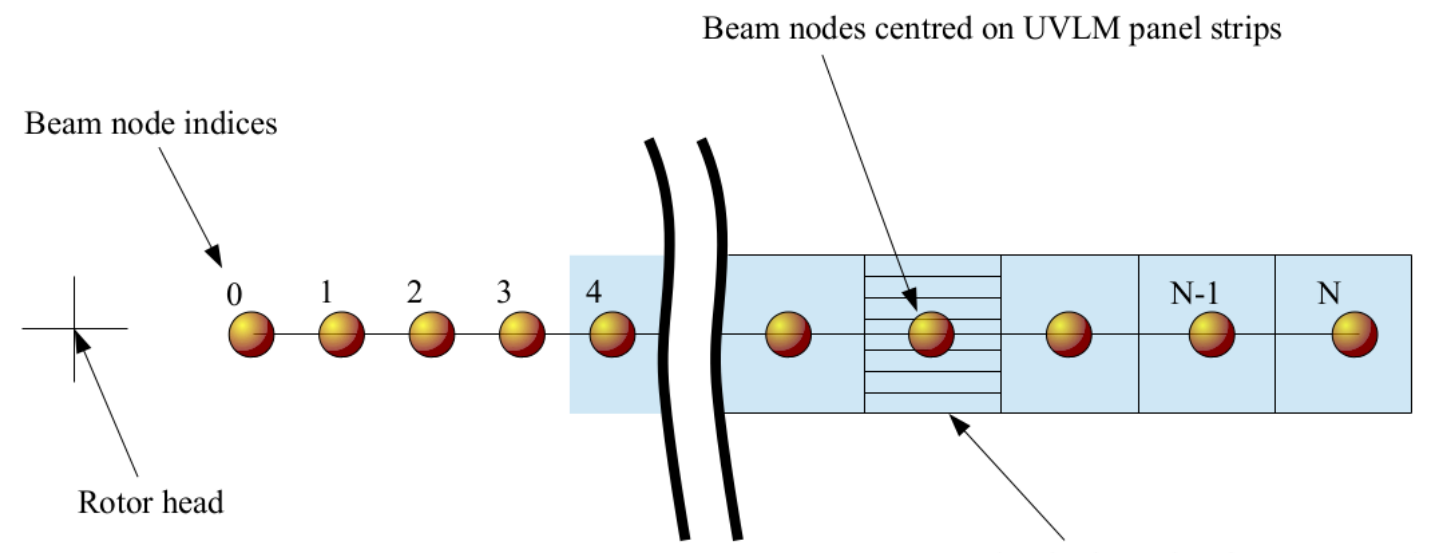

Chord-wise strip of UVLM panels

Fig. 8 Structural nodes and aerodynamic panels used in the Flexit simulations

\section{Twist deformation}

For the purpose of quantitative comparison, we use the torsional twist angle at the tip of each blade as a convenient metric. This quantity is the sum of the twist at each finite element node accumulated from the root to the tip.

Figure 11 presents the tip twist history of the 10 degree blade root twist angle case for two values of the twist angular velocity damping coefficient $K_{T}$. This illustrates the sensitivity of the tip twist deformation angle to the value of the damping coefficient $K_{T}$. Since the thrust coefficient is expected to be linearly dependent on the twist angle, this highlights the importance of estimating torsional (and bending) damping correctly in predicting the dynamics of an aeroelastic rotor system. See section VIIIf for more details.

Figure 12 presents the twist deformation of the blades at the end of the simulation for the three blade root twist angle cases respectively. Note that a positive twist deformation corresponds to a leading edge pitch-up deformation. The majority of the twist deformations take place at node 2 in the location of the Flexbeam where the torsional stiffness is designed to be very low.

\section{Blade deflections}

The predicted blade tip deflection as a function of time (frame number) for the simulations of 8,10 and 12 degree blade root twist angle cases is presented in figure 13 for blade 1. An Euler-Bernoulli beam bending velocity-dependent damping coefficient $\beta$ value of 20 was used.

In preliminary work, we considered simplifying the Flexit simulations by ignoring the Flexbeam (the part of the beam inboard of the blade aerodynamic profile). Figure 14 presents a comparison of the flapping behaviour with and without the Flexbeam for the 12 degree blade root twist angle case for blade 1.

Three conclusions can be drawn from figure 14.

1) We see that the magnitude of steady-state tip deflection of the "with" Flexbeam case is roughly twice that of the "without" Flexbeam deflection case.

2) We see that the frequency of the first flapping mode of the "with" Flexbeam case is approximately two thirds of the first flapping mode of the "without" Flexbeam case. This is due to the larger span of the beam in the "with" Flexbeam case.

3) We see that there is a distinct change in beam tangent angle at approximately 1.1 metres span in the "with" Flexbeam case. We attribute this to a local reduction in flapping stiffness at this radius that has been incorporated into the Flexbeam design expressly to allow large bending deformations to take place there. The simulation results confirm that bending does take place in the beam inboard of the 1.1 metre radius, but the deformations are very small and are difficult to see in the figure.

Figure 15 presents the deformations for each of blades of the 8 degree blade root twist angle case along side the tip deflection histories. 

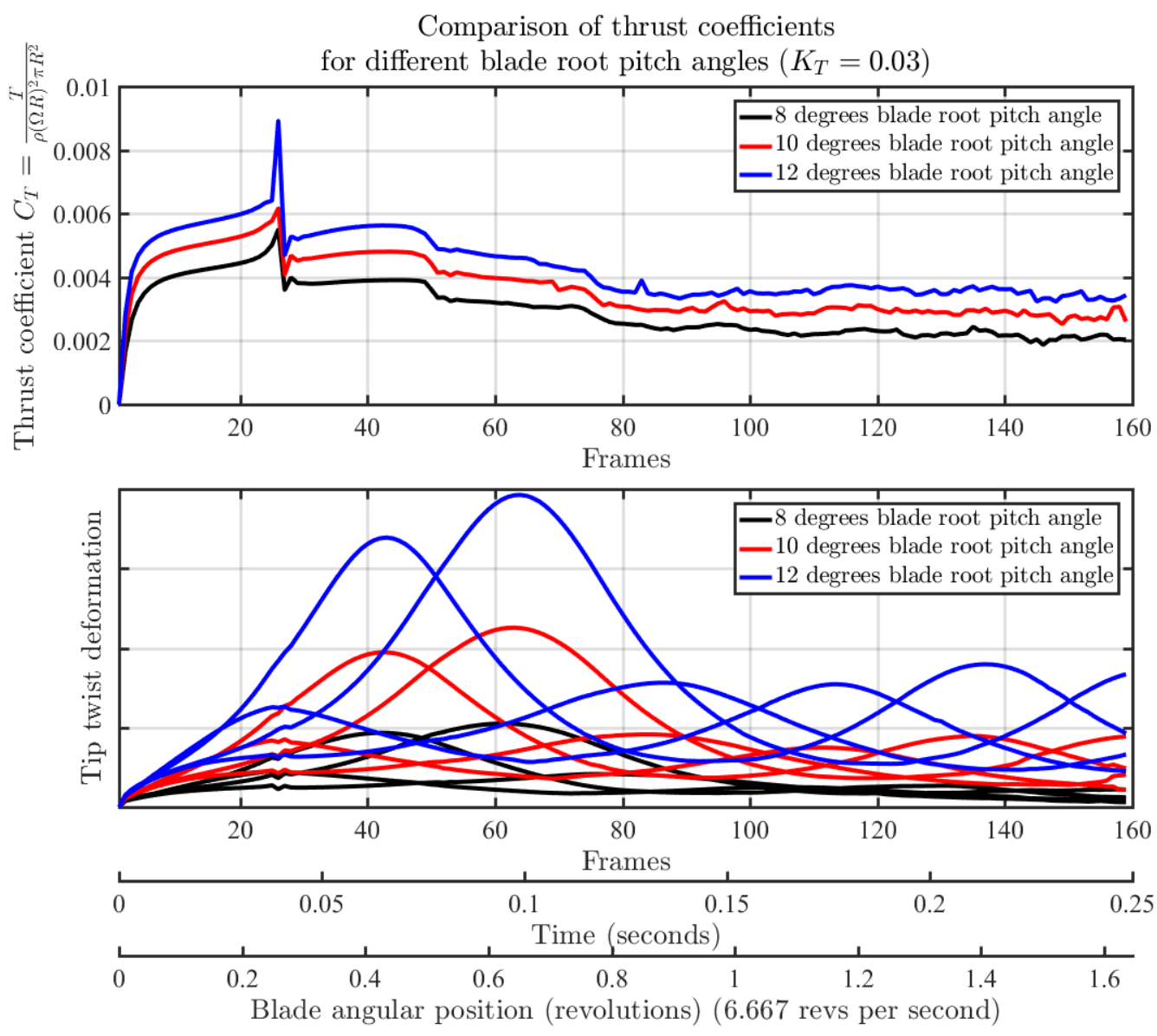

Fig. 9 Thrust coefficient predictions for a range of blade root twist angles $\left(K_{T}=0.03\right)$

\section{E. Wake and down-wash geometry}

A feature of the Flexit program graphical user interface is that the free wake geometry evolution with time can be displayed. A snapshot of the wake geometry from the 10 degree blade root twist case at frame 160 (the end of the simulation) is shown in Figure 16. The vortices shed from the tips and the roots of each blade are clearly visible. The downwash velocities are in general agreement with actuator disk theory predictions.

\section{Comparison and validation}

\section{A. Comparison with commercial Navier-Stokes Solver}

In order to gain confidence in the results predicted by Flexit, comparisons with a commercial Navier-Stokes (NS) solver were performed. The NS simulations considered an H135 helicopter at 1000ft in undisturbed air in a hover condition. The aerodynamic forces and moments were calculated for the blade aerodynamic profile. These simulations were performed using the density based (coupled) finite volume solver in Ansys Fluent (version 17.2) (see reference [14]). It uses an unstructured mesh 3D Navier-Stokes equation solver with cell-centred finite-volume spatial discretization. To model the flow-field and associated forces, steady state Reynolds Averaged Navier-Stokes (RANS) equations were solved. The two equation $k-\omega$ SST model by Menter (see reference [15]) was used for modelling the turbulence. The continuity and the conservation equations were solved using the implicit formulation in a coupled manner, while the turbulence equations were solved in a segregated manner. To calculate the convective flux, a Roe Flux Difference Splitting (Roe-FDS) scheme was used (see reference [16]). For spatial discretization, a second order upwind scheme was used for the flow equations, while a first order upwind scheme was used for the turbulence equations. The 


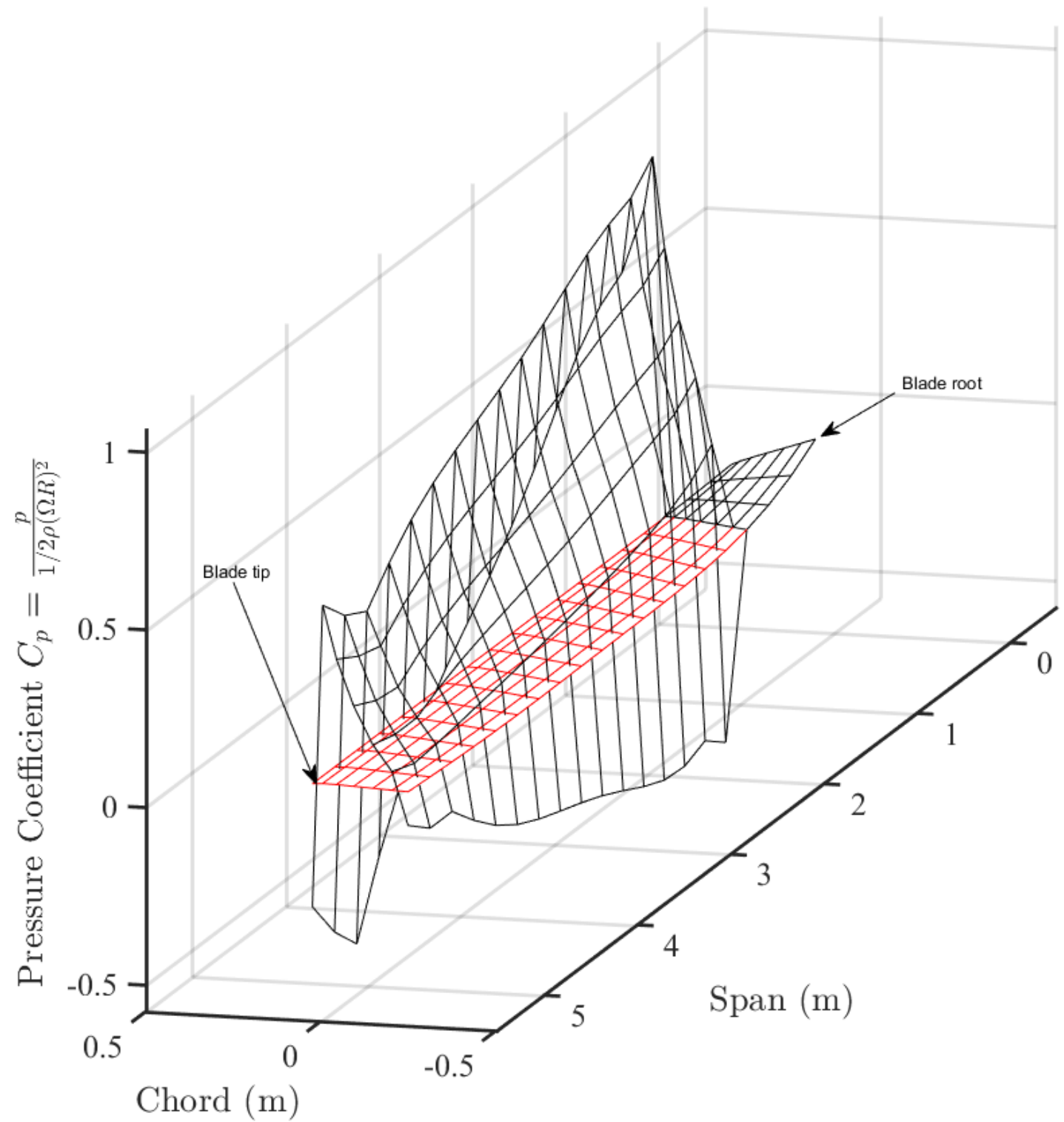

Fig. 10 Blade pressure coefficient distribution for the 8 degree blade root twist angle case

gradients were calculated using the Least Squares Cell-based approach (see reference [17]). These equations were solved in a domain with linear dimension measuring ten times the rotor diameter, which contained the whole helicopter. The domain was discretized with 22.5 million mixed-type finite volume elements (quads, tetras, prism etc.), mostly concentrated near the helicopter. Wall boundary conditions were imposed on the helicopter body and the rotor blade surfaces, and far-field boundary conditions were imposed on the domain boundaries. The predicted wall $Y+$ value was less than 80 over the wall surfaces. A Moving Reference Frame (MRF) modelling approach was used to model the effect of main rotor blade rotation on the flow field, while the tail rotor was not modelled (see reference [17]). The air was modelled as an ideal gas, and Sutherland relation for viscosity was used. Other reference air properties were taken from the US Standard Atmosphere data tables. The convergence criteria to determine steady solution was chosen as a $10^{-3}$ drop in magnitude of the normalised residuals and the stabilisation of the forces and the velocity flow-field. The rotor blades were assumed to be rigid, with a constant blade root pitch angle as the rotor blade changes its position with 
Twist deformation

Comparison of blades for 10 degree root pitch angle

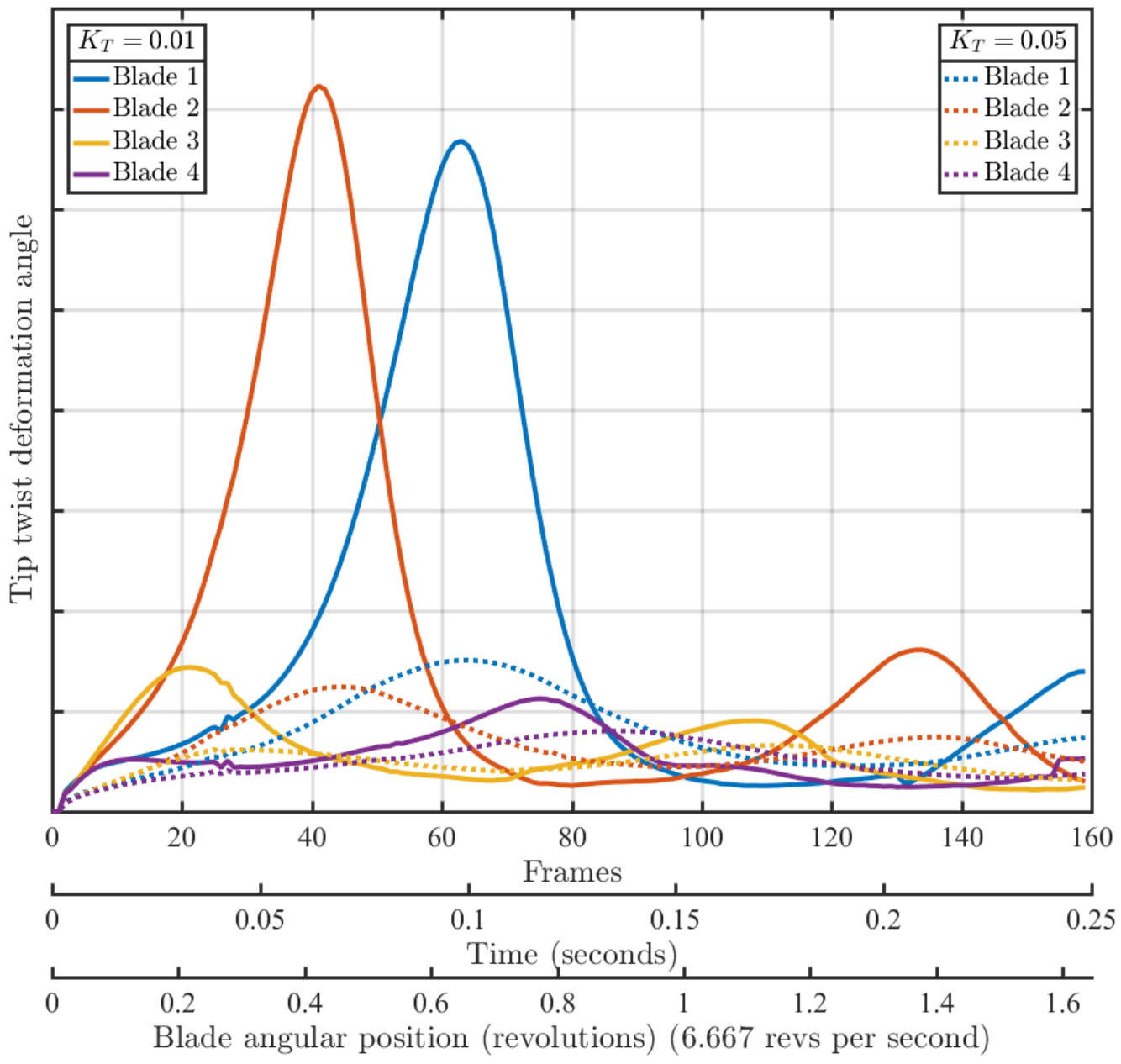

Fig. 11 Blade tip deflection predictions for high and low twist damping coefficient

respect to azimuth angle. The obtained results are only valid for the four rotor blade positions, as shown in figure 17

The modelling performed with Fluent did not take account of structural deformation. For this reason we compare the Fluent results with those of Flexit when the structural deformation in Flexit has been disabled. Under these conditions, we can be certain that the aerodynamic geometry modelled in Flexit is generally equivalent to that modelled in Fluent. There are still certain specific discrepancies in conditions between the two sets of modelling runs. For example, the Fluent modelling takes into account fluid shear stress forces associated with viscous flow and the presence of the fuselage and tail boom, while Flexit does not. On the other hand Fluent ignores the presence of the Flexbeam that acts as an end-plate to the inboard end of each blade and suppresses the formation of inboard tip vortices. Figure 18 presents comparisons of pressure coefficients predicted by Fluent and by Flexit for the 8 degree blade root twist case at a range of span-wise positions. Over the mid-section of the span there is good agreement between the Fluent and Flexit predictions of $C_{p}$. However, Fluent predicts an increase in the pressure coefficient near the leading edge of the blade that is not present in the Flexit results. This trend increases at the blade tip.

Table 1 presents a complete set of thrust coefficient data, and includes a column containing the thrust coefficients predicted by the Fluent CFD simulations. The lower $C_{p}$ values predicted by Flexit go some way to explaining why the Flexit $C_{T}$ values in the table are lower than those of Fluent. 

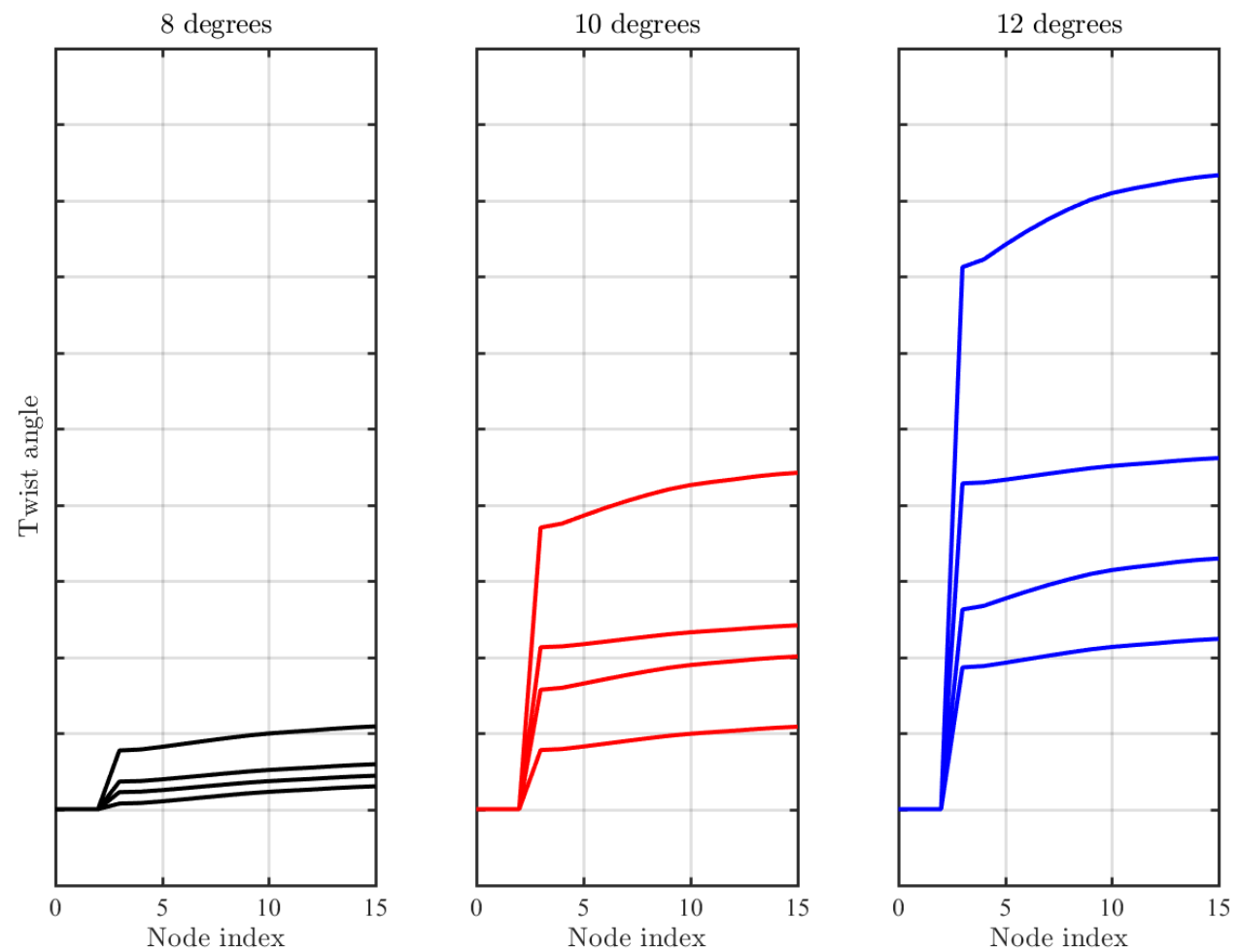

Fig. 12 Twist deformation distribution for all blade root twist angles $\left(K_{T}=0.03\right)$

\begin{tabular}{|cccc|}
\hline Case & Blade root angle (degs.) & Flexit $C_{T}$ & Fluent $C_{T}$ \\
\hline \hline 1 & 8 & 0.0020 & 0.00333 \\
2 & 10 & 0.0028 & 0.00475 \\
3 & 12 & 0.0036 & 0.00624 \\
\hline
\end{tabular}

Table 1 Thrust coefficient predictions

\section{B. Comparison with fluid-structure interaction modelling}

Flexit was also compared with a fluid-structure interaction (FSI) analysis, which describes the response of a structure to the aerodynamic forces. The FSI approach used a very loosely coupled framework where the aerodynamic load and the structural response were obtained using two independent codes. The aerodynamic load was calculated with Fluent (see section $\mathrm{A}$ ) and is coupled with a reduced-order finite element (FE) structural rotor blade model using Nastran. Material and cross sectional properties for the blade were derived from data provided by AHD. Due to the very long simulation time of Fluent (approximately 120 hours on a 64-node cluster) a one-way coupling was considered, which means that no feedback was provided from the deformed structure to recalculate the induced aerodynamic loads. This methodology is presented in the flow chart shown in figure 19

The aerodynamic, as well as centrifugal and inertial loadings were applied to the FE structural nodes in order to calculate the resulting deformation. Figure 20 presents comparisons of the blade tip deflections predicted by Flexit (using a flapping damping coefficient $\beta$ value of 20) and those predicted by the FSI simulation work for the 8, 10 and 12 degree blade root twist cases.

The initial amplitudes and phases compare well, but the Flexit amplitudes damp out more quickly. The Flexit predictions exhibit a large variation between blades that is not present in the FSI data. It is to be expected that there will be little or no variation in the deformation of individual blades in the FSI scheme since there is no feedback between 
Tip deflection of blade 1

Comparison for a range of blade root pitch angles, $\beta=20$

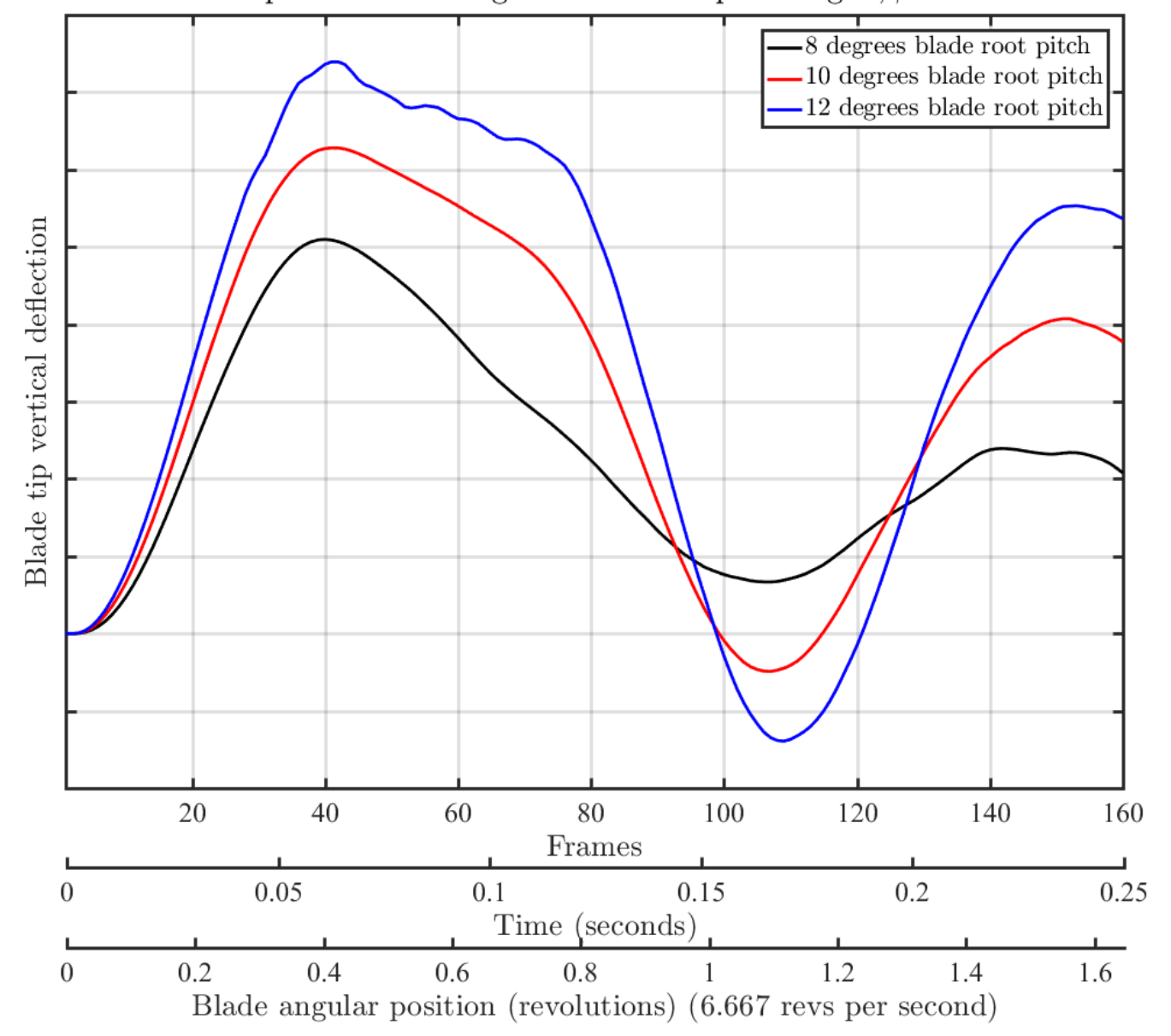

Fig. 13 Blade tip deflection predictions for a range of blade root twist angle

deformation and aerodynamic loads.

\section{Conclusions and future work}

A new aeroelastic simulation framework called Flexit is being developed in order to analyse the dynamic behaviour of flexible structures under aerodynamic, elastic and inertial loading. Due to the implementation of NVIDIA CUDA technology fast computational times are achieved. Fast execution combined with simple input data makes Flexit particularly well suited for use in the early design stages. The speed of the computation means that a large number of alternative designs can be analysed in a short period of time. This in turn allows the aerospace designer to explore large design spaces and minimises the risk of carrying designs with undesirable behaviour forward to detailed design stages.

While the Flexit data presented in this paper indicates expected trends, the values of predicted thrust coefficient are significantly under-estimated when compared with both rigid geometry viscous flow predictions and full scale experimental data. The reason for this under-prediction compared with the Fluent predictions can largely be explained by comparing the chord-wise distributions of pressure coefficient presented in figure 18 The Fluent $C_{p}$ distributions show a significant leading edge suction, and this phenomenon is not modelled by the UVLM in Flexit. Another reason for the low thrust coefficient predictions of Flexit may be due to low local inflow velocity direction vectors due to the wake downwash predicted by Flexit. We intend to investigate whether the inflow velocity directions used by Fluent result in higher angles of attack.

The blade flapping deformation results from the Flexit framework are shown to be in general agreement with the 
H135 Cambered blades, 12 degree blade root pitch angle. With and without FlexBeam. Frame:120
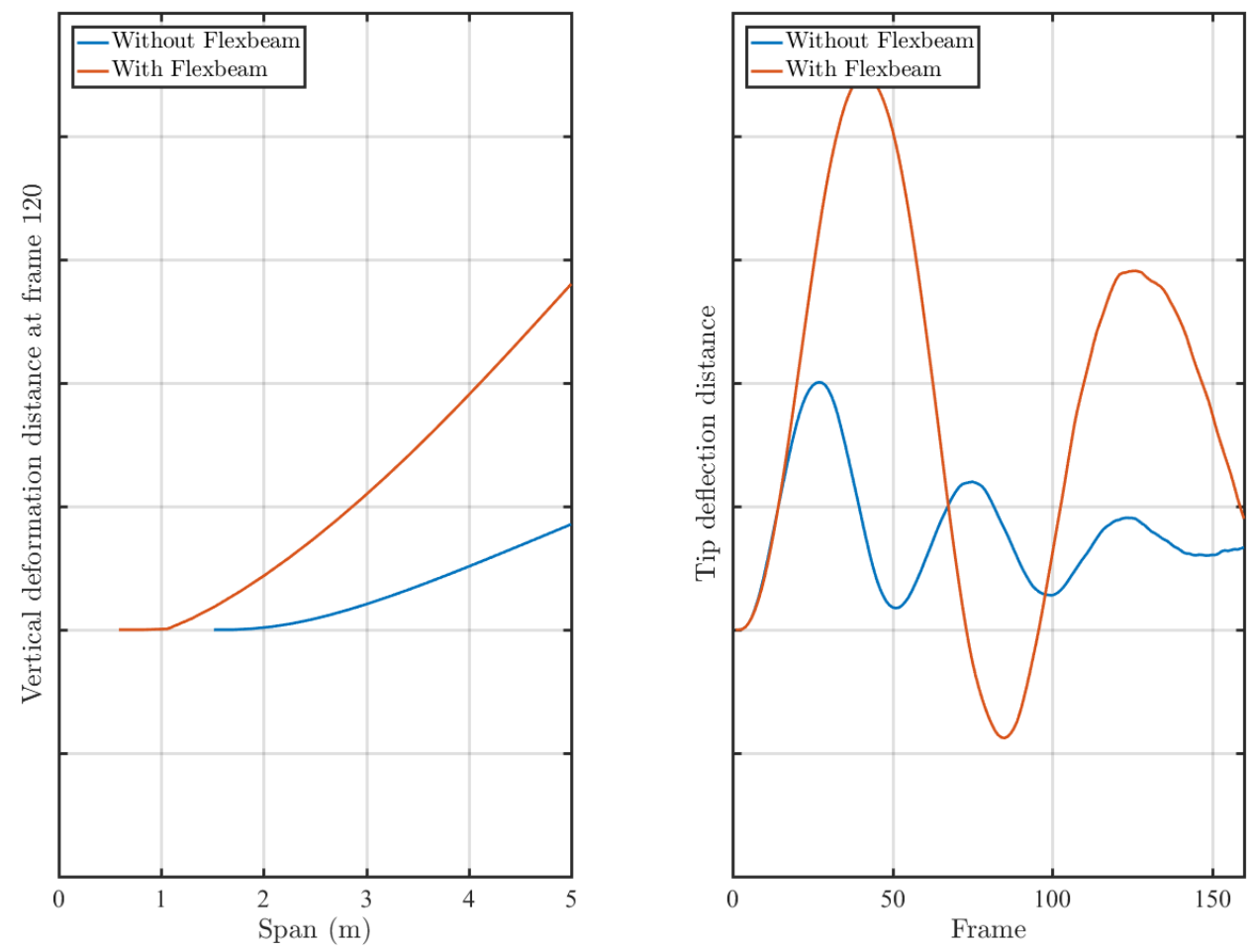

Fig. 14 Comparison of flapping deflection predictions with and without the Flexbeam

results of Nastran-based FSI computations. This agreement is despite the fact that while Flexit incorporates a loose coupling feedback between aerodynamic pressure loading and flapping deformation, the Nastran-based FSI modelling does not.

The most notable differences between the results can be summarised as

1) approximately $30 \%$ under-estimation of the thrust coefficient compared to both Fluent/FSI computations and full-scale data,

2) the flapping amplitude predicted by Flexit damps out much faster than the flapping predicted by the FSI,

3) the Fluent simulations predict a significant leading edge suction that is not modelled by the Flexit simulations (because the leading edge radius is ignored by the camber line surfaces used in Flexit.

In the future we hope to continue work on the Flexit framework, and topics for further development associated with the modelling of the physics and the mathematical formulations include

1) comparison of blade in-flow velocity vectors predicted by Flexit versus those predicted by Fluent,

2) comparison of predictions from Flexit with data from full scale experimental tests when full scale data becomes available,

3) running of simulations using Rankine vortex formulations to compare the results with those using Biot-Savart vortex formulations,

4) attempting to obtain accurate design data for the position of the elastic centre relative to the blade chord. If this data can be obtained, then we will perform simulation runs with these new elastic centre positions and compare the twist deformations predicted in these new runs with the existing results,

5) implementation of beam modelling using a finite difference scheme for the Timoshenko coupled PDEs. For the relatively small deformations predicted for the H135 rotor disk we anticipate that predictions using the Timoshenko scheme will be very similar to those of the Euler-Bernoulli scheme. For designs with much larger deformations (leading to more non-linear effects) we expect the Timoshenko approach to give more accurate results,

6) further investigation of the values of the torsional damping $\left(K_{T}\right)$ and bending damping $(\beta)$. In particular we 
Deflection for H135 Cambered blades

8 degree blade root pitch angle
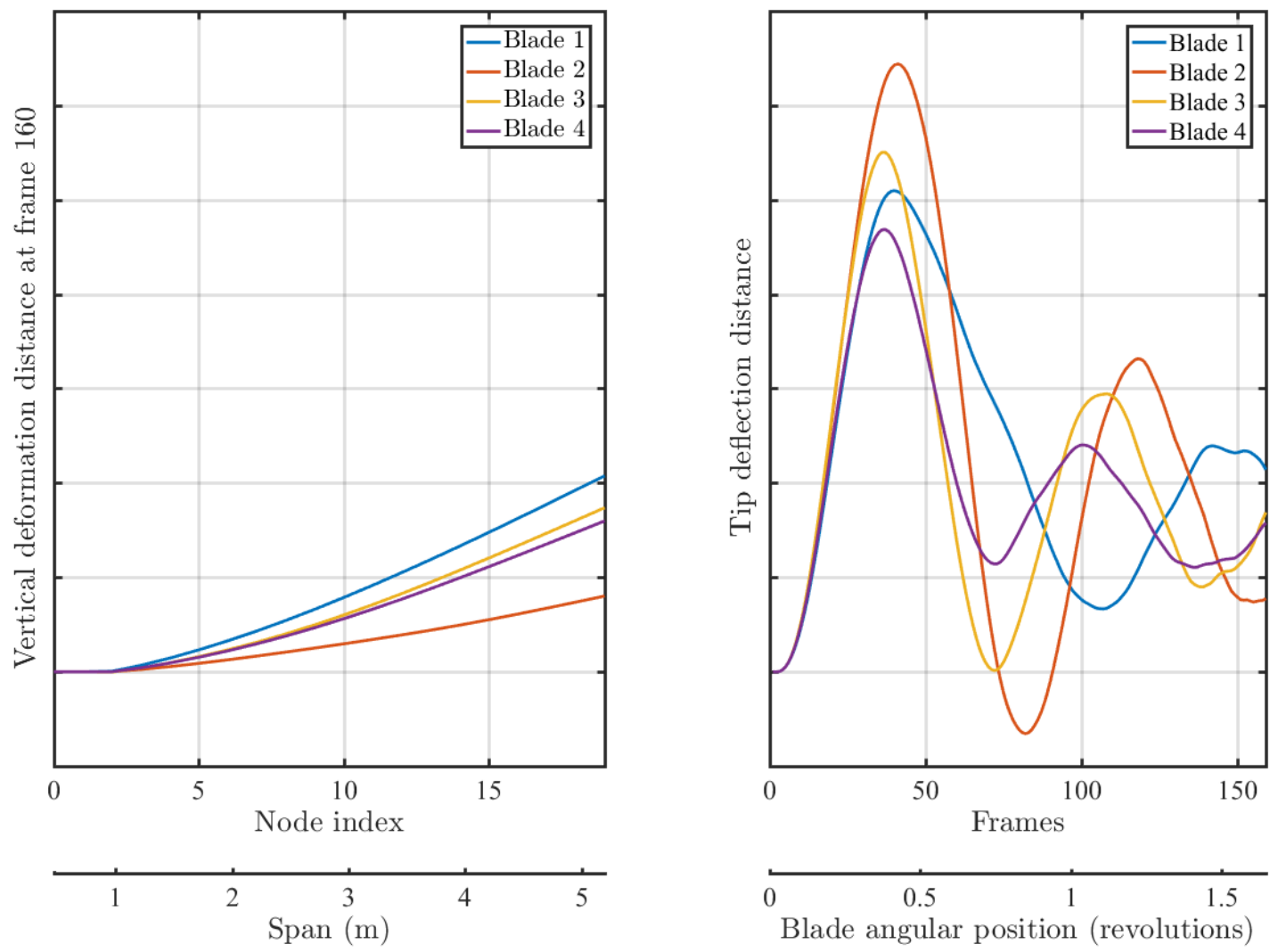

Fig. 15 Blade flapping deformation predictions with tip deflection histories for the 8 degree blade root twist angle case

wish to a) define the relation between the bending damping $\beta$ and Rayleigh damping which is often used in other structural dynamic theory and b) investigate how to accurately estimate the values of $K_{T}$ and $\beta$, since these constants have a large effect on the amplitude of dynamic deformations.

Topics associated with the computational performance include:

1) working on improving computational efficiency by exploiting multiple GPUs when they are present and by parallelizing some of the remaining serial parts of the algorithms,

2) investigating the cause of the step increase in computation time experienced in many of the simulation runs at around frame 155. If the cause can be found, then we will attempt to remove the problem,

3 ) attempting to improve the memory utilisation of the Flexit program. The modelling of the four main rotor blades for approximately 200 frames requires approximately 2GBytes of computer memory which is near the limit of the address space for the 32 bit application on a Windows desktop machine. To perform longer simulations we will consider changing the software configurations in order to use all the available physical memory,

4) investigate how to improve the computational performance by exploiting various geometric symmetries in the lifting surface configurations. For example to exploit the four-fold circumferential symmetry found in the H135 helicopter rotor disk in the hover.

\section{Acknowledgements}

The authors would like to acknowledge the support of Airbus Helicopters UK and Airbus Helicopters Deutschland, as well as ATI and Innovate UK. The work was carried out as part of the BladeSense project. 


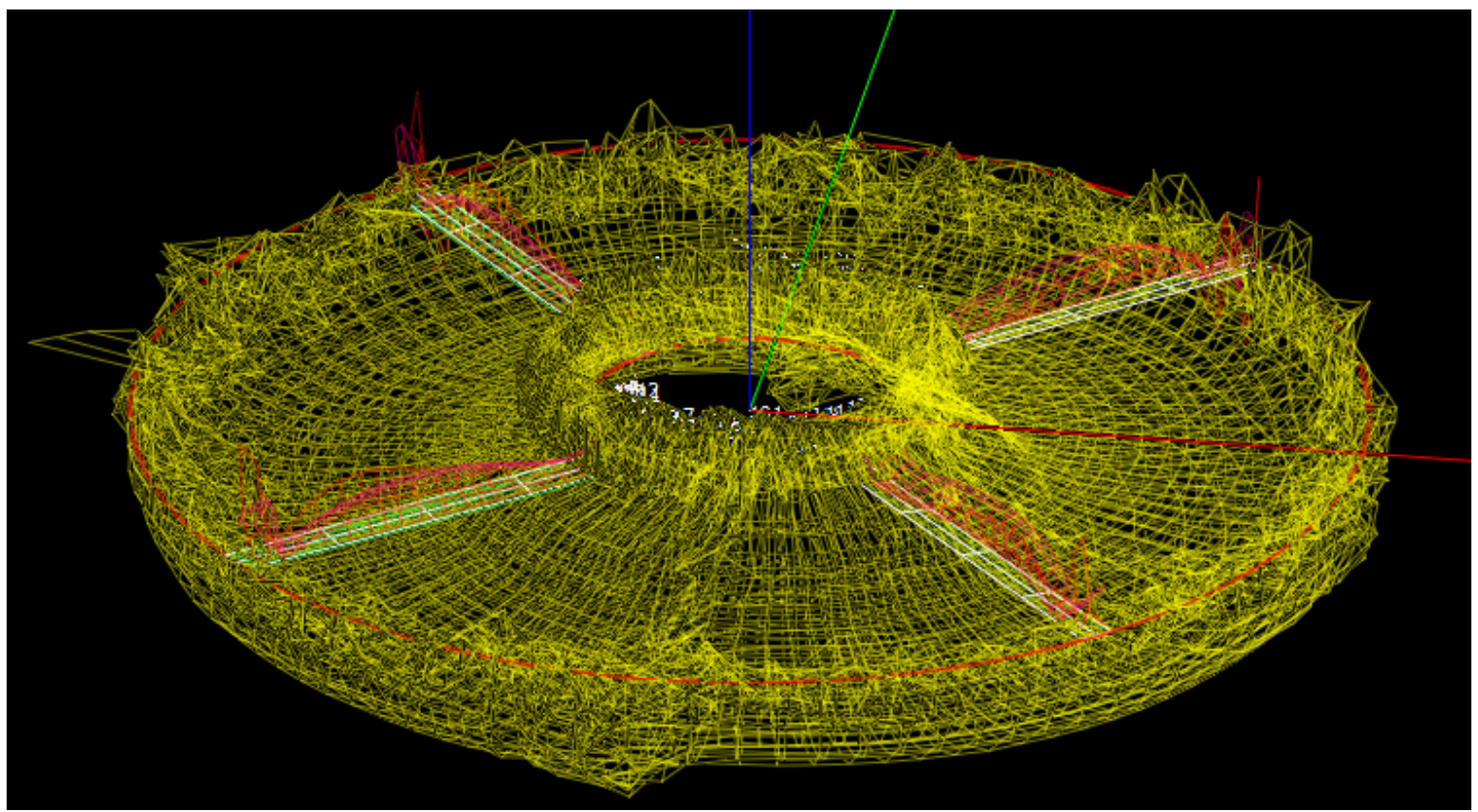

Fig. 16 Wake geometry predictions for the four-bladed configuration

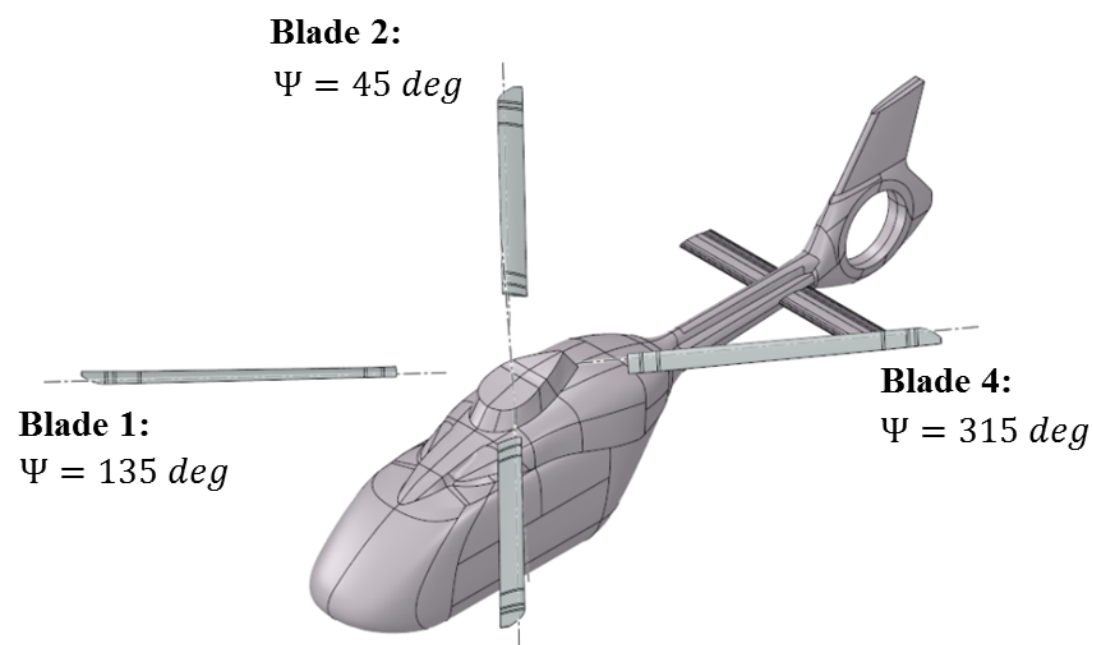

Blade 3:

$\Psi=225 \mathrm{deg}$

Fig. 17 Four rotor blade azimuth location used for CFD calculation 

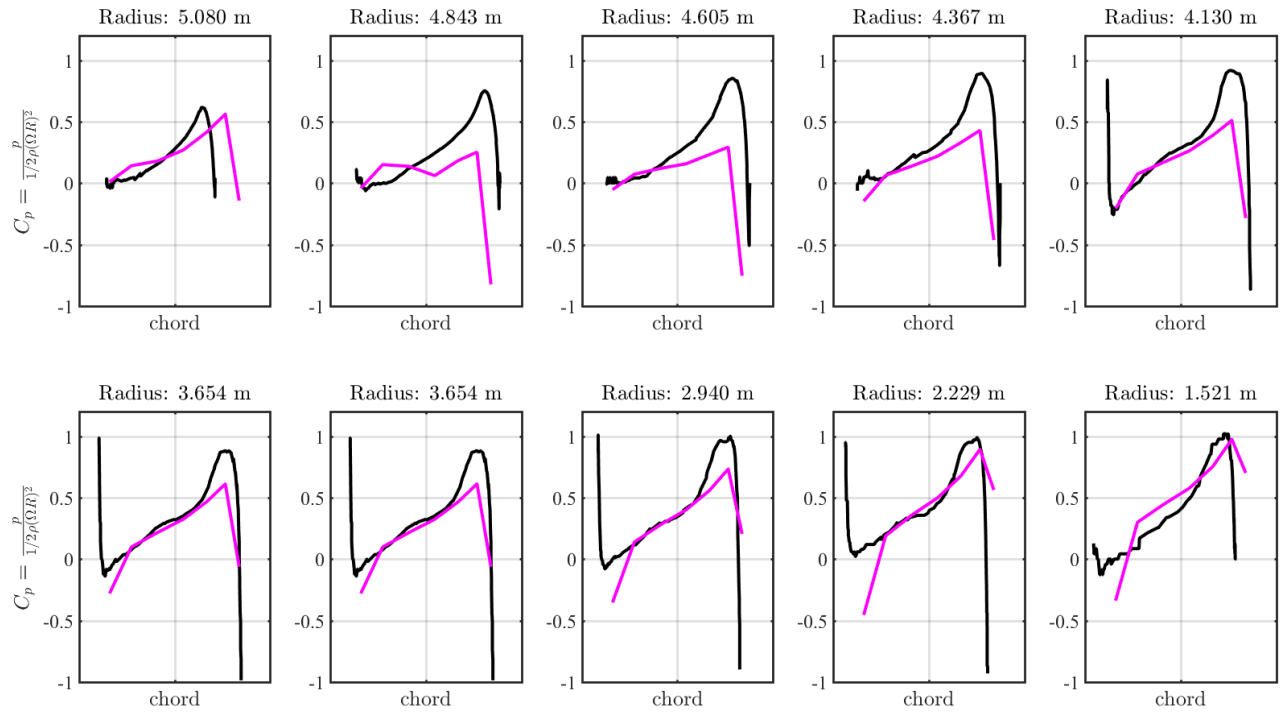

Fig. 18 Blade section pressure coefficient distribution comparison for the 8 degree blade root twist angle case

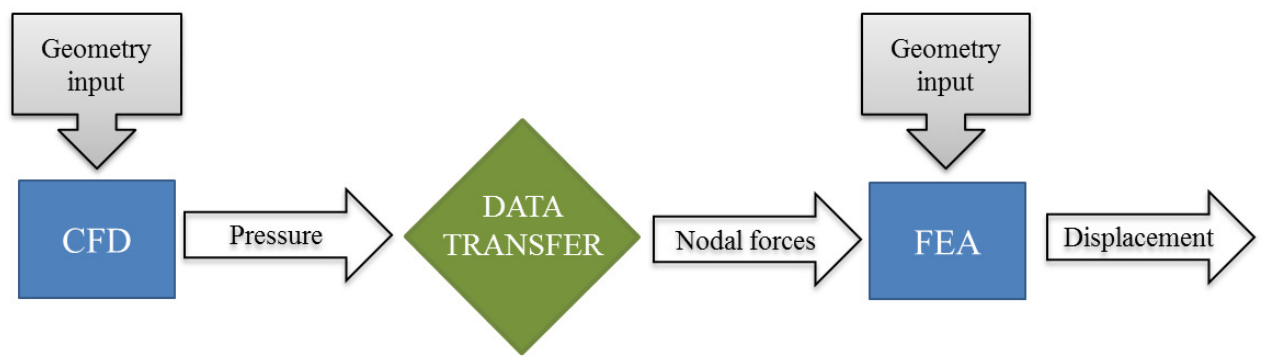

Fig. 19 Flow chart demonstrating the FSI process of the loose coupling between the CFD and FEA tool

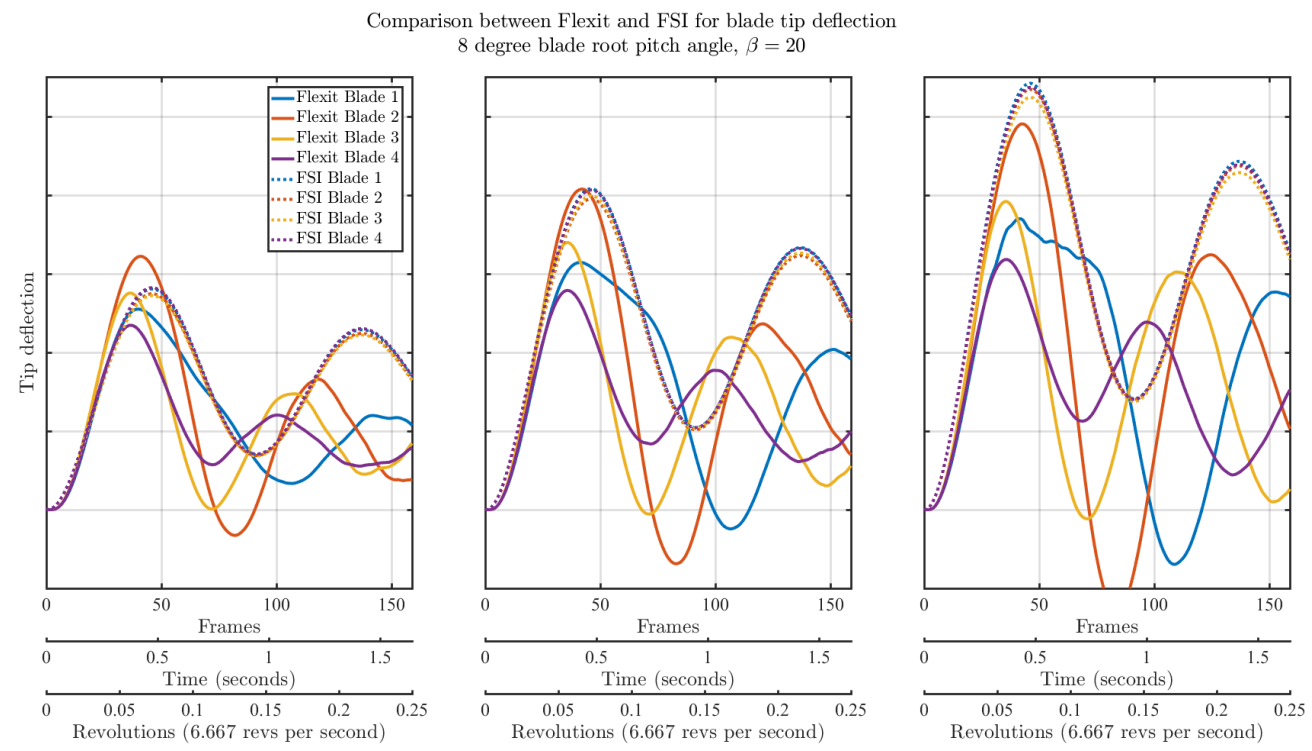

Fig. 20 Blade tip flapping deflection comparison between Flexit predictions and FSI predictions, $\beta=20$. 


\section{References}

[1] Katz, J., Plotkin, A., Low Speed Aerodynamics, Cambridge University Press, 2001.

[2] Murua, J., Palacios, R., and Graham, J. M. R., "Applications of the unsteady vortex-lattice method in aircraft aeroelasticity and flight dynamics,", 2012.

[3] Drela, M., Flight Vehicle Aerodynamics, MIT Press, 2014.

[4] Hazebrouck, G. A., "OpenVogel Open source software for aerodynamics,", 2017. URL https://sites.google.com/ site/gahvogel/

[5] Zhu, Q., Wolfgang, M., Yue, D., and Triantafyllou, M., "Three- dimensional flow structures and vorticity control in fish-like swimming," Journal of Fluid Mechanics, Vol. 468, 2002, pp. 1-28.

[6] Izraelevitz, J. S., Zhu, Q., and Triantafyllou, M. S., "State-Space Adaptation of Unsteady Lifting Line Theory: Twisting/Flapping Wings of Finite-Span," AIAA Journal, Vol. 55, No. 4, 2016.

[7] Portapas, V., Cooke, A., and Lone, M., "Modelling framework for flight dynamics of flexible aircraft," Aviation, Vol. 20, No. 4, 2016, pp. 173-182.

[8] “Official NVIDIA CUDA Web site,”, 2017. URL http://www.nvidia.com/object/cuda_home_new.html]

[9] Kundu, B., and Ganguli, R., "Analysis of weak solution of Euler-Bernoulli beam with axial force," Applied Mathematics and Computation, Vol. 286, 2016, pp. 247-260.

[10] "Airbus Helicopters on-line specification for the H135 helicopter,", 2017. URL http://airbushelicoptersinc.com/ products/H135-specifications.asp

[11] Bansemir, H., Emmerling, S., "Fatigue Substantiation and Damage Tolerance Evaluation of Fiber Composite Helicopter Components," RTO AVT Specialists' Meeting on "Application of Damage Tolerance Principles for Improved Airoworthiness of Rotorcraft", 1999.

[12] Kampa, K., Enenkl, B., Polz, G., and Roth, G., "Aeromechanic aspects in the design of the EC135," 23rd European Rotorcraft Forum, Dresden, Germany, 1997.

[13] Padfield, G. D., Helicopter Flight Dynamics, $2^{\text {nd }}$ ed., Blackwell Publishing, 2007.

[14] Ansys Fluent User's Guide v17.2, Ansys, 2016.

[15] Menter, F. R., "Two-equation eddy-viscosity turbulence models for engineering applications," American Institute of Aeronautics and Astronautics Journal, Vol. 32, No. 8, 1994, pp. 1598-1605.

[16] Roe, P. L., "Characteristic-Based Schemes for the Euler Equations," Annual Review of Fluid Mechanics, Vol. 18, No. 1, 1986, pp. 337-365.

[17] Ansys Fluent Theory Guide v17.2, Ansys, 2016. 
2018-01-13

Fast computational aeroelastic analysis of helicopter rotor blades

Fleischmann, Dominique

AIAA

Fleischmann D, Weber S, Lone MM, Fast computational aeroelastic analysis of helicopter rotor blades, Proceedings of 2018 AIAA Aerospace Sciences Meeting, 8-12 January 2018,

Kissimmee, Florida, USA, Paper AIAA 2018-1044

http://dx.doi.org/10.2514/6.2018-1044

Downloaded from Cranfield Library Services E-Repository 